\title{
Identification of differentially expressed genes and preliminary validations in cardiac pathological remodeling induced by transverse aortic constriction
}

\author{
HUI-BO WANG ${ }^{1-3^{*}}$, RONG HUANG ${ }^{1-3^{*}}$, KANG YANG $^{4}$, MAN XU $^{1-3}$, DI FAN ${ }^{1-3}$, MING-XIN LIU ${ }^{1-3}$, \\ SI-HUI HUANG ${ }^{1-3}$, LI-BO LIU ${ }^{1-3}$, HAI-MING WU ${ }^{1-3}$ and QI-ZHU TANG ${ }^{1-3}$ \\ ${ }^{1}$ Department of Cardiology, Renmin Hospital of Wuhan University; ${ }^{2}$ Cardiovascular Research Institute of Wuhan University; \\ ${ }^{3}$ Hubei Key Laboratory of Cardiology; ${ }^{4}$ Department of Urology, Renmin Hospital of Wuhan University, \\ Wuhan, Hubei 430060, P.R. China
}

Received January 26, 2019; Accepted July 9, 2019

DOI: $10.3892 / \mathrm{ijmm} .2019 .4291$

\begin{abstract}
Cardiac remodeling predisposes to heart failure if the burden is unresolved, and heart failure is an important cause of mortality in humans. The aim of the present study was to identify the key genes involved in cardiac pathological remodeling induced by pressure overload. Gene expression profiles of the GSE5500, GSE18224, GSE36074 and GSE56348 datasets were downloaded from the Gene Expression Omnibus database. Differentially expressed genes (DEGs), defined as $\mid \log _{2} \mathrm{FCl}>1$ ( $F C$, fold change) and an adjusted $\mathrm{P}$-value of $<0.05$, were screened using the R software with the limma package. Gene ontology enrichment analysis was performed and a protein-protein interaction (PPI) network of the DEGs was constructed. A cardiac remodeling model induced by transverse aortic constriction (TAC) was established. Furthermore, consistent DEGs were further validated using reverse transcription-quantitative polymerase chain reaction (RT-PCR) analysis, western blotting and immunohistochemistry in the ventricular tissue samples after TAC or sham operation. A total of 24 common DEGs were identified (23 significantly upregulated and 1 downregulated), of which 9 genes had been previously confirmed to be directly involved in cardiac remodeling. Hence, the level of expression of the other 15 genes was detected in subsequent studies via RT-PCR. Based on the results of the PPI network analysis and RT-PCR, we further detected the protein levels of Itgbll and Asporin, which were consistent with the results of bioinformatics
\end{abstract}

Correspondence to: Dr Qi-Zhu Tang, Department of Cardiology, Renmin Hospital of Wuhan University, 238 Jiefang Road, Wuhan, Hubei 430060, P.R. China

E-mail: qztang@whu.edu.cn

${ }^{*}$ Contributed equally

Key words: cardiac remodeling, heart failure, pressure overload, bioinformatics, differentially expressed genes, functional enrichment analysis, protein-protein interaction analysis and RT-PCR. The expression of Itgbl1, Aspn, Fstl1, Mfap5, Col8a1, Ltbp2, Mfap4, Pamr1, Cnksr1, Aqp8, Meox1, Gdf15 and Srpx was found to be upregulated in a mouse model of cardiac remodeling, while that of Retnla was downregulated. Therefore, the present study identified the key genes implicated in cardiac remodeling, aiming to provide new insight into the underlying mechanism.

\section{Introduction}

The prevalence and mortality of cardiovascular disease (CVD) have exceeded those of malignant tumors, making it the most important threat to human health and quality of life worldwide (1). Patients with CVD are at increased risk of developing end-stage heart failure (HF). With the rapid progress in medical research, the understanding of the etiology and pathogenesis of HF has gradually improved. However, there is currently no effective treatment for end-stage HF. Therefore, the prevention and treatment of HF are the main areas of research in the field of CVD (2). Cardiac remodeling is characterized by an increased burden in CVD and is regulated by various cytokines and neurohumoral factors. The heart has an adaptive compensatory mechanism for maintaining cardiac structure and function. Such compensation usually appears as an increase in the thickness, weight and capacity of the ventricle, as well as microscopic changes in terms of hypertrophy of the cardiac myocytes, increased myocardial fibrosis and interstitial cell proliferation, among others. Long-term persistence of these burdens results in decompensation and, eventually, $\operatorname{HF}(3,4)$.

Genes are the functional units of DNA, which store all the information necessary for the reproduction and functioning of all forms of life (5). Gene expression refers to the process of protein synthesis under the guidance of genes. Several gene expression changes are involved in the occurrence and development of various diseases (6). The gene chip, also referred to as the DNA chip or DNA microarray, uses the principle of hybridization to detect the existence and quantity of the corresponding fragments for the functional and genomic research of genes; it has been widely used in scientific research and for the clinical diagnosis of various diseases, including CVD $(6,7)$. 
Successful establishment of animal models is the most critical step in the study of the pathophysiology and molecular mechanisms underlying myocardial remodeling. Common models of cardiac remodeling are established by injection of catecholamines, such as isoproterenol, phenylephrine and angiotensin II, as well as by transverse aortic constriction (TAC) in spontaneously hypertensive rats. TAC increases the afterload by constricting the aorta and increasing the aortic pressure (8). In the present study, we downloaded and analyzed four original microarray datasets in the Gene Expression Omnibus (GEO) database (https://www.ncbi.nlm.nih.gov/geo), namely GSE5500, GSE18224, GSE36074 and GSE56348, in order to explore the potential mechanisms underlying cardiac pathological remodeling induced by TAC-mediated pressure overload. The correlation between cardiac pathological remodeling and differentially expressed genes (DEGs) was filtered using the limma package of $\mathrm{R}$ software ( $\mathrm{R}$ version 3.3.3). We subsequently identified numerous potential DEGs related to cardiac pathological remodeling and explored their function via Gene Ontology (GO) and Kyoto Encyclopedia of Genes and Genomes (KEGG) pathway enrichment analyses using the Database for Annotation, Visualization and Integrated Discovery (DAVID) online tool, version 6.8 (https://david. ncifcrf.gov/home.jsp). Finally, a mouse model of cardiac remodeling induced by TAC was established, and reverse transcription-quantitative polymerase chain reaction (RT-PCR) analysis was used to verify the expression of DEGs, thereby providing new insights into potential molecular targets for the treatment of pathological cardiac remodeling.

\section{Materials and methods}

Microarray data information and identification of DEGs. GEO is a free public repository of gene microarray data (http://www.ncbi.nlm.nih. gov/geo/) (9), from which the gene expression profiles of GSE5500, GSE18224, GSE36074 and GSE56348 datasets were determined in the left ventricular (LV) myocardial tissue of TAC or sham-operated mice. GSE5500 included 6 TAC and 4 sham-operated LV myocardial tissue samples (submission date: August 10, 2006) (10); GSE18224 included 8 TAC and 8 sham-operated LV myocardial tissue samples (submission date: September 23, 2009) (11); GSE36074 included 14 TAC and 5 sham-operated LV myocardial tissue samples (submission date: February 24, 2012) (12); and GSE56348 included 5 pairs of TAC and sham-operated LV myocardial tissue samples (submission date: March 29, 2014) (13). The GSE5500, GSE18224 and GSE36074 data were based on the GPL1261 platforms (Affymetrix Mouse Genome 4302.0 Array, Affymetrix; Thermo Fisher Scientific, Inc.); the GSE56348 data were based on the GPL6480 platforms (Affymetrix Mouse Gene 1.0 ST Array) (Table I).

Raw data (TXT format) of genomic expression were integrated for analysis, and data preprocessing, including background correction, normalization, logarithmic conversion and screening of DEGs were subsequently performed using $\mathrm{R}$ software with the limma package. Statistically significant DEGs were defined as $\log _{2} \mathrm{FC}>1$ or $\log _{2} \mathrm{FC}<-1$ (FC, fold change) and an adjusted P-value of $<0.05$. Volcano plots and heatmaps of these screened DEGs were constructed using $R$ software.
GO terms and pathway enrichment analysis. DAVID version 6.8 is an open-source website for scientists to explore the biological functions of DEGs (14). GO analysis was conducted using the sub-databases GOTERM_BP_DIRECT, GOTERM_CC_ DIRECT and GOTERM_MF_DIRECT, and KEGG pathway analysis was conducted using DAVID version 6.8 to analyze the DEGs at the functional level. $\mathrm{P}<0.01$ was set as the cut-off indicating statistically significant differences.

Integration of protein-protein interaction (PPI) network and identification of hub genes. PPIs include direct physical interactions and indirect functions (15). DEGs were selected from the four data series (i.e., GSE5500, GSE18224, GSE36074 and GSE56348), and a PPI network was constructed. The online database STRING (http://string-db.org) was used to construct the PPI network and analyze the functional interactions based on the above screened DEG-encoded proteins. The hub genes were screened by calculating the number of interconnections. Proteins located at the center nodes may be considered as core proteins with important physiological functions, and the corresponding gene is a key candidate gene.

Animals and animal models. All animal care and experimental procedures were approved by the Animal Care and Use Committee of Renmin Hospital of Wuhan University (Wuhan, China), and conformed to the Guidelines for the Care and Use of Laboratory Animals published by the United States National Institutes of Health (16). Adult male specific pathogen-free (SPF) grade C57BL/6 mice $(n=40,8-10$ weeks old, weighing 23.5-25.5 g) and adult male and female SPF grade Sprague-Dawley (SD) rats were purchased from the Institute of Laboratory Animal Science, Chinese Academy of Medical Sciences, Beijing, China. The TAC model was selected mainly because of the TAC model of original chip data analysis. In the study of TAC-mediated cardiac remodeling, the most commonly used time points are 4 and 8 weeks. Previous studies have reported that significant changes in cardiac remodeling and decreased cardiac function are observed 4 weeks after TAC, whereas at 8 weeks after TAC, end-stage HF is more likely $(17,18)$. Furthermore, most of the original chip data we analyzed were samples analyzed for $\sim 4$ weeks. Therefore, testing at 4 weeks after TAC was selected. The mice were randomly divided into two groups (TAC, $n=25$; sham, $n=15$ ) and allowed to acclimatize for 7 days prior to the experiments. The animals were anesthetized with $80 \mathrm{mg} / \mathrm{kg}$ sodium pentobarbital (Sigma-Aldrich; Merck KGaA) by intraperitoneal injection, and subsequently subjected to TAC or sham operation, as described previously (17). A total of 6 mice in the TAC group and 3 mice in the sham group died during or after the operation. The animals were euthanized via $200 \mathrm{mg} / \mathrm{kg}$ sodium pentobarbital by intraperitoneal injection.

Echocardiographic measurement. At 4 weeks after the operation, echocardiography was used to measure the LV structure and function in mice that were anesthetized by inhalation of $1.5 \%$ isoflurane. Echocardiography was performed using a MyLab 30CV system (Esaote SpA) equipped with a $10-\mathrm{MHz}$ linear array ultrasound transducer, as previously described (18). Two-dimensional images were captured from the LV parasternal long axis and parasternal short axis at the 
Table I. GEO Datasets used for the bioinformatics analysis.

\begin{tabular}{lllllrrr}
\hline Study & Genus & Tissue & GEO & Platform & Sham & TAC & (Refs.) \\
\hline Bisping et al & Mus musculus & Left ventricular & GSE5500 & GPL1261 & 4 & 6 & $(10)$ \\
Fliegner et al & Mus musculus & Left ventricular & GSE18224 & GPL1261 & 8 & 8 & $(11)$ \\
Skrbic et al & Mus musculus & Left ventricular & GSE36074 & GPL1261 & 5 & 14 & $(12)$ \\
Lai et al & Mus musculus & Left ventricular & GSE56348 & GPL6246 & 10 & 10 & $(13)$ \\
\hline
\end{tabular}

GEO, Gene Expression Omnibus; TAC, transverse aortic constriction.

level close to the papillary muscles at a frame rate of $120 \mathrm{~Hz}$. LV end-systolic diameter (LVESD), LV end-diastolic dimension (LVEDD), LV ejection fraction (EF) and LV fractional shortening (FS) were obtained by LV M-mode tracing with a sweep speed of $50 \mathrm{~mm} / \mathrm{sec}$. LVESD, LVEDD, EF and FS were analyzed for $>10$ beats per heart.

Histological analysis. Following completion of echocardiographic and hemodynamic measurements, the mice were immediately euthanized. Body weight, heart and lung weight, and tibial length were measured for each mouse. Heart weight/body weight (HW/BW, mg/g), lung weight/body weight (LW/BW, mg/g) and heart weight/tibial length (HW/TL, mg/mm) ratios in each group were calculated using these data. The heart was harvested and immediately placed in $10 \% \mathrm{KCl}$ solution to induce diastolic arrest. Subsequently, the hearts were fixed in $4 \%$ paraformaldehyde for 3 days. Fixed myocardial tissues were dehydrated, embedded in paraffin, and cut into 5- $\mu \mathrm{m}$ sections as described previously (19). Sections from the middle segment of each heart were subjected to hematoxylin and eosin (H\&E) staining for the observation of overall morphology.

For immunohistochemistry, fixation, embedding and sectioning of cardiac specimens were performed in a similar manner as for H\&E staining. The heart sections were heated for antigen retrieval using a pressure cooker. The sections were incubated with anti-integrin subunit beta-like 1 (Itgbl1; Santa Cruz Biotechnology, Inc.; sc-365162, 1:50) and anti-Asporin (Abcam; ab58741, 1:100), and developed using peroxidase-coupled secondary antibodies and 3,3'-diaminobenzidine as a substrate. Images were captured at a magnification of x400 from 10 fields/heart.

$R T-q P C R$. The mRNA expression level of the DEGs with research value was measured using qPCR, as described previously (19). Total RNA was isolated from LV myocardium using TRIzol reagent (Invitrogen; Thermo Fisher Scientific, Inc.) and mRNA was reverse-transcribed to cDNA using oligo(dT) primers. Gene expression levels were analyzed using the Light Cycler 480 Q-PCR System and Light Cycler 480 SYBR-Green 1 Master Mix (Roche Diagnostics). Target gene mRNA expression was normalized to the internal control glyceraldehyde-3-phosphate dehydrogenase (GAPDH). The forward and reverse primer pairs used for RT-PCR are shown in Table II.

Western blotting. The protein expression level was measured by western blotting as described previously. The cardiac LV tissue was lysed in RIPA lysis buffer, and the protein concentration was measured using the BCA Protein Assay Kit (Thermo Fisher Scientific, Inc.; 23227). Protein lysates $(50 \mu \mathrm{g})$ were electrophoresed on $10 \%$ sodium dodecyl sulfate-polyacrylamide gels, and subsequently transferred onto polyvinylidene fluoride (PVDF) membranes (EMD Millipore; IPFL00010) by a gel transfer device. The PVDF membranes were blocked with 5\% non-fat milk for $1 \mathrm{~h}$ and incubated with primary antibodies against Itgbll (Santa Cruz Biotechnology, Inc.; sc-365162, 1:200) and Asporin (Abcam, ab58741, 1:400), overnight at $4^{\circ} \mathrm{C}$. After washing three times with Tris-buffered saline containing Tween 20, the membrane was stained with anti-mouse or anti-rabbit $\operatorname{IgG}(1: 10,000)$ for $1 \mathrm{~h}$. The blots were scanned and analyzed using an Odyssey infrared imaging system (LI-COR Biosciences). Specific protein expression levels were normalized to GAPDH (Cell Signaling Technology, Inc.; 5174, 1:2,000).

Cultured neonatal rat ventricular myocytes (CMs) and cardiac fibroblasts (CFs). Neonatal rat CMs and CFs were prepared based on our published literature $(20,21)$. Briefly, adequate neonatal hearts from 1- to 2-day-old Sprague-Dawley (SD) rats were cut into $\sim 1-\mathrm{mm}^{3}$ pieces and digested in enzyme solutions. Isolation of CMs and CFs was performed by differential time adhesion. BrdU (0.1 mM) was used to inhibit the proliferation of the CFs present in the CM fraction. The CMs and CFs were cultured in DMEM/F12 containing fetal bovine serum (15\% for CMs or $10 \%$ for CFs), streptomycin $(100 \mathrm{mg} / \mathrm{ml})$, and penicillin $(100 \mathrm{IU} / \mathrm{ml})$ at $37^{\circ} \mathrm{C}$ in a humidified incubator with $5 \% \mathrm{CO}_{2}$. CMs and CFs were stimulated by angiotensin (Ang) II $(1 \mu \mathrm{M})$ and transforming growth factor (TGF) $\beta(10 \mathrm{ng} / \mathrm{ml})$, respectively. The effect of stimulation was evaluated by immunofluorescence staining. The CM fraction was detected with anti- $\alpha$-actinin staining. CFs were stained with anti- $\alpha$-smooth muscle actin (SMA) to observe fluorescence intensity.

Immunofluorescence staining. Immunofluorescence staining was performed as previously described (17). Briefly, cell coverslips were washed three times with PBS, fixed with $4 \%$ formaldehyde, permeabilized in $0.2 \%$ Triton X-100, and stained with anti- $\alpha$-actinin (1:100 in $1 \%$ goat serum) or anti- $\alpha$-SMA (1:100 in $1 \%$ goat serum) overnight after blocking with $10 \%$ goat serum for $60 \mathrm{~min}$ at $37^{\circ} \mathrm{C}$. The coverslips were then incubated with Alexa Fluor 488-goat anti-rabbit secondary antibody for $60 \mathrm{~min}$ at $37^{\circ} \mathrm{C}$, with DAPI (Invitrogen; Thermo Fisher Scientific, Inc.; S36939) for nuclear staining. Images were captured by a special Olympus DX51 fluorescence microscope (Olympus Corporation). 
Table II. Forward and reverse primer pairs.

\begin{tabular}{lll}
\hline Gene names & \multicolumn{1}{c}{ Forward } & Reverse \\
\hline Mfap4 & GCAACCCCTGGACTGTGATG & TTGTCATGTCGCAGAAGACGG \\
Ltbp2 & AACAGCACCAACCACTGTATC & CCTGGCATTCTGAGGGTCAAA \\
Retnla & CCAATCCAGCTAACTATCCCTCC & ACCCAGTAGCAGTCATCCCA \\
Cthrc1 & TGGACCAAGGAAGCCCTGAGT & TGAACAGGTGCCGACCCAGA \\
Pamr1 & ATGGAGCTAGACAGATGGGC & GACCGTGTACTCTCTTGGCAA \\
Itgbl1 & GTGGAAACTGTTACTGCGAGG & TGGCAAGAACATTCACCACATAC \\
MFAP5 & GTCTTGGCAATCAGCATCCC & CCAGATTAGGGTCGTCTGTGAAT \\
Meox1 & GAAACCCCCACTCAGAAGATAGC & TCGTTGAAGATTCGCTCAGTC \\
Aqp8 & TGTGTAGTATGGACCTACCTGAG & ACCGATAGACATCCGATGAAGAT \\
Aspn & AAGGAGTATGTGATGCTACTGCT & ACATTGGCACCCAAATGGACA \\
Srpx2 & ATGGTACGCAGGCTCAGGTTA & TGAGTAGCATGTGGCTTCTCC \\
Col8a & AGGAGAAGTACCGTTAGCCAG & TACCGGGCTTTCCAATTCCTG \\
Cilp & ATGGCAGCAATCAAGACTTGG & AGGCTGGACTCTTCTCACTGA \\
Frzb & CACAGCACCCAGGCTAACG & TGCGTACATTGCACAGAGGAA \\
Fstl1 & CACGGCGAGGAGGAACCTA & TCTTGCCATTACTGCCACACA \\
\hline
\end{tabular}

Statistical analysis. Data are presented as the mean \pm standard error of the mean from at least six independent experiments. To determine statistical significance, the results were compared using Student's two-tailed t-tests. All statistical analyses were performed using GraphPad Prism 5.0 software (GraphPad Software, Inc.). A P-value of $<0.05$ at a $95 \%$ confidence level was considered to indicate statistically significant differences.

\section{Results}

Identification of DEGs. As shown in Table I, 4 datasets (GSE5500, GSE18224, GSE36074 and GSE56348) were included in the present study. Following data normalization and DEG analysis using $\mid \log _{2} \mathrm{FCl}>1$ and $\mathrm{P}<0.05$ as cut-offs, a total of 188, 293, 253 and 67 DEGs were identified from the GSE5500, GSE18224, GSE36074 and GSE56348 datasets, respectively. The volcano plots and heatmaps of the first 50 DEGs are displayed in Fig. 1. In bioinformatics, the method of collecting and intersecting multiple profile datasets is commonly used. The selection criterion of 'all four positive' methods is commonly used and the results obtained are considered as reliable. After using the Venny website (http://bioinfogp.cnb.csic.es/tools/venny/index.html) for integrated bioinformatics, a total of 24 consistent DEGs were identified from the four profile datasets (Fig. 2), including 23 upregulated and 1 downregulated genes in the mouse model of cardiac remodeling induced by TAC vs. sham operation (Fig. 2A, Table III).

DEG GO analysis in TAC-induced cardiac remodeling. To explore the biological function of DEGs in a mouse model of TAC-mediated pressure overload-induced LV remodeling, the 24 screened DEGs were uploaded to DAVID version 6.8. The DEGs were found to be enriched in biological processes, including extracellular fibril organization, cell adhesion, wound healing, elastic fiber assembly, and negative regulation of cell growth and cartilage development. For cell component, the
Table III. DEGs identified from the four profiles $(n=24)$.

\begin{tabular}{ll}
\hline DEGs & \multicolumn{1}{c}{ Genes } \\
\hline Upregulated & Ltbp2, Postn, Cilp, Mfap4, Thbs4, Col8a1, \\
& Timp1, Nppa, Gdf15, Pamr1, Lox, Acta1, \\
& Itgbl1, Ctgf, Cnksr1, Frzb, Mfap5, Meox1, \\
& Tgfb2, Aqp8, Fst11, Aspn, Srpx2 \\
Downregulated & Retnla
\end{tabular}

DEGs, differentially expressed genes.

DEGs were mainly enriched in extracellular region, proteinaceous extracellular matrix, extracellular space, extracellular matrix, basement membrane, and extracellular exosome. Molecular function analysis revealed that the upregulated DEGs were mainly enriched in growth factor activity, heparin binding, and calcium ion binding (Fig. 2B and Table IV). No DEGs were found to be significantly enriched in KEGG pathway enrichment analysis.

Integration of PPI network and identification of hub genes. The 24 screened DEGs from these four datasets were selected to construct a PPI network using the STRING online database. A total of 18 DEGs (all upregulated) of the 24 commonly altered DEGs were filtered into the DEG PPI network complex. These 18 DEGs included (in descending order) Postn, Lox, Ctgf, Itgbl1, Aspn, Fstl1, Mfap5, Acta1, Nppa, Tgfb2, Timp1, Col8a1, Ltbp2, Mfap4, Thbs4, Cilp, Pamr1 and Frzb sequentially (Fig. 3).

Establishment of a cardiac pathological remodeling model in mice. For 4 weeks after TAC, the remodeling responses of these mice in response to pressure overload were detected. TAC mice exhibited a significant cardiac pathological remodeling 
A

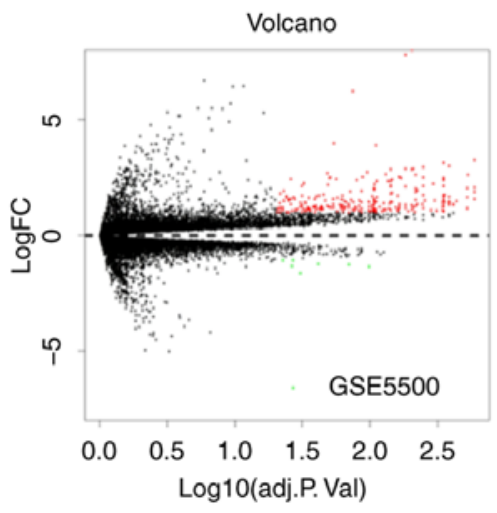

C
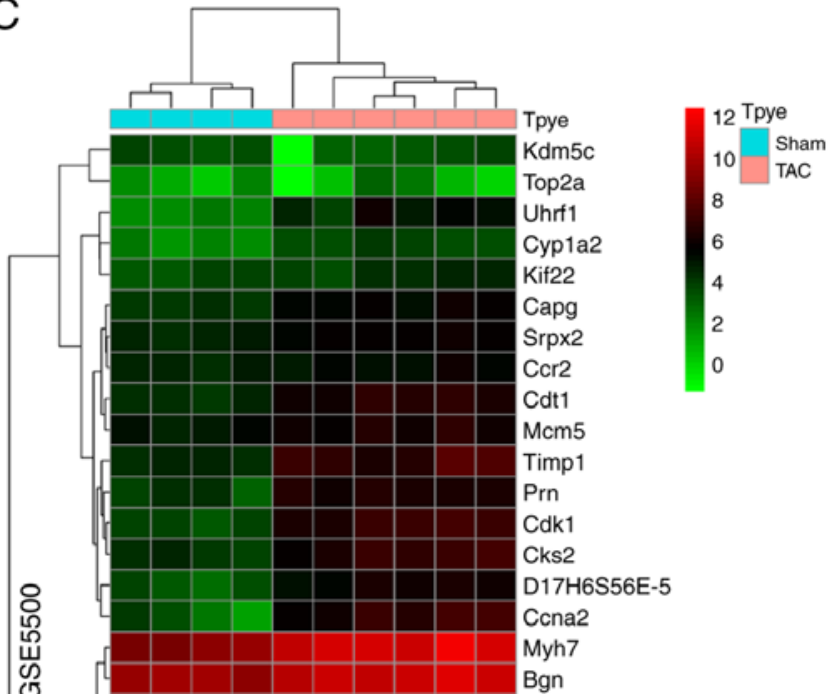

.
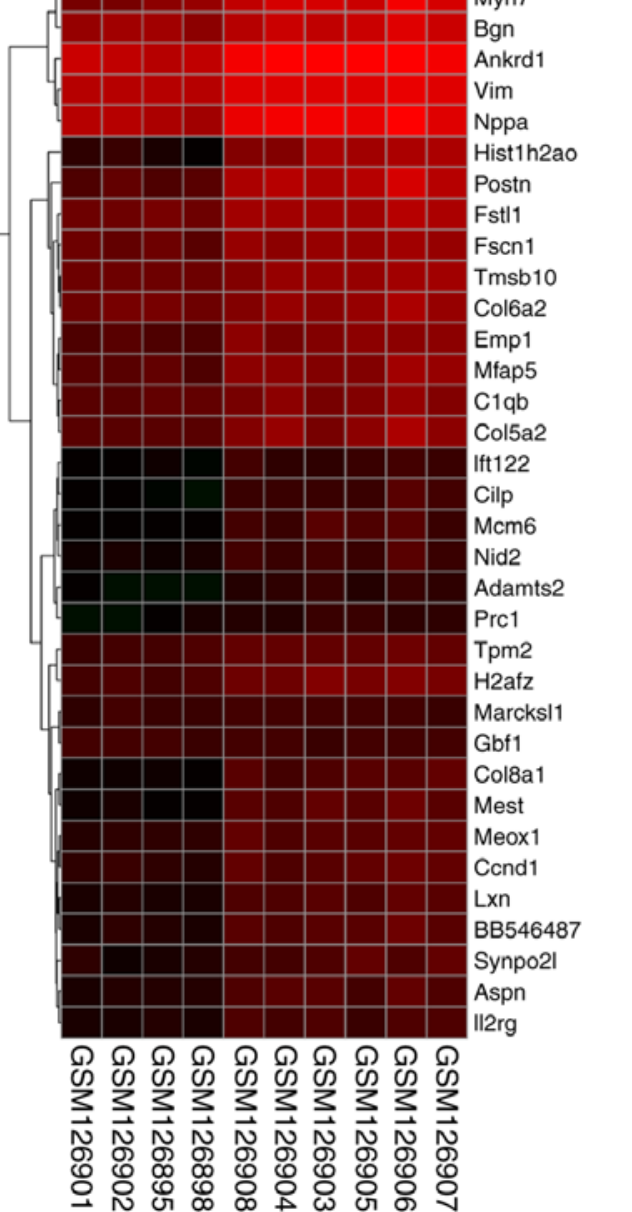

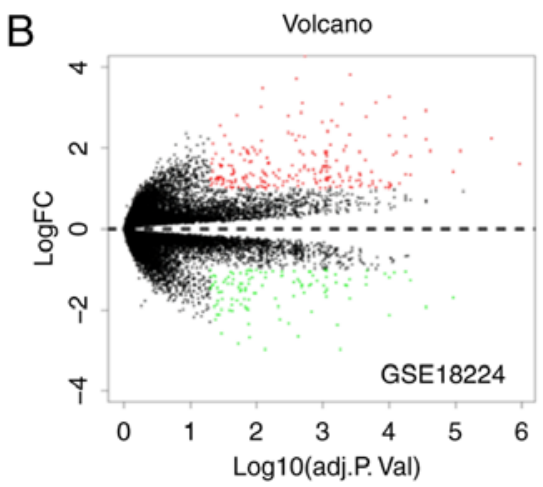

D

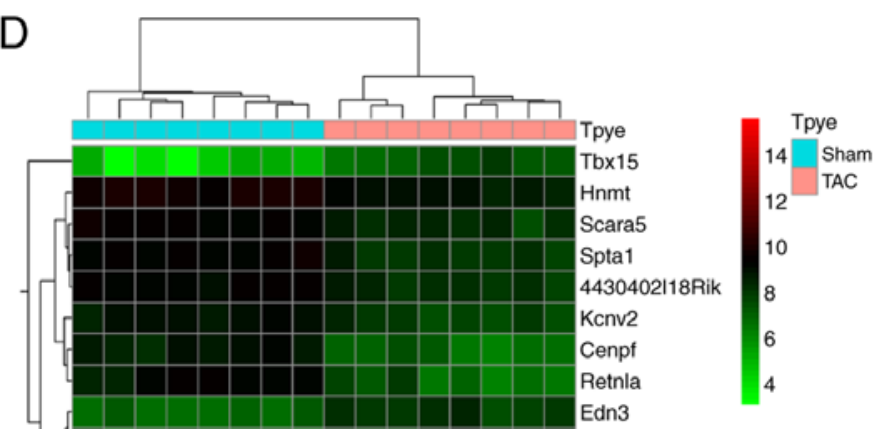

Edn

A730054J21Rik 1500009L16Rik Nirc3

Critt1

Ltbp2

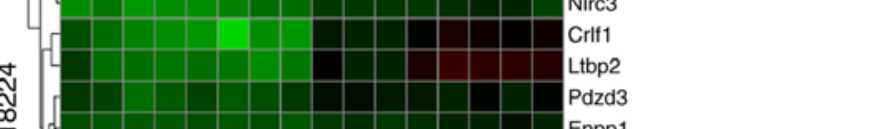

今

Hspa1I

Gnao Milt11

Gck Comtd1 Aqp8 Frzb Acta1 Nppa Ankrd23 Ban Myot Pcolce Synpo21 Rcan1 Cilp Col8a1 Flnc Fbn1 Loxl1 Cmss1 Lgals4 Ano10 Thbs 4 Cnksr-1 Itgbl1 Pmepa1 Haus8 Prg4 Mybpc2 Plekho1 Cyr61

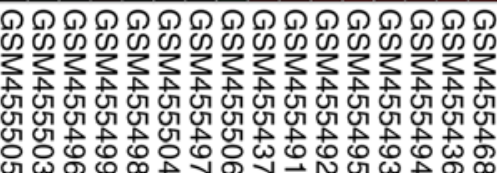

Figure 1. Volcano plot and heatmaps of differentially expressed genes (DEGs): (A) Volcano plot of DEGs for dataset GSE5500; (B) volcano plot of DEGs for dataset GSE18224; (C) heatmaps of DEGs for dataset GSE5500; (D) heatmaps of DEGs for dataset GSE18224. 

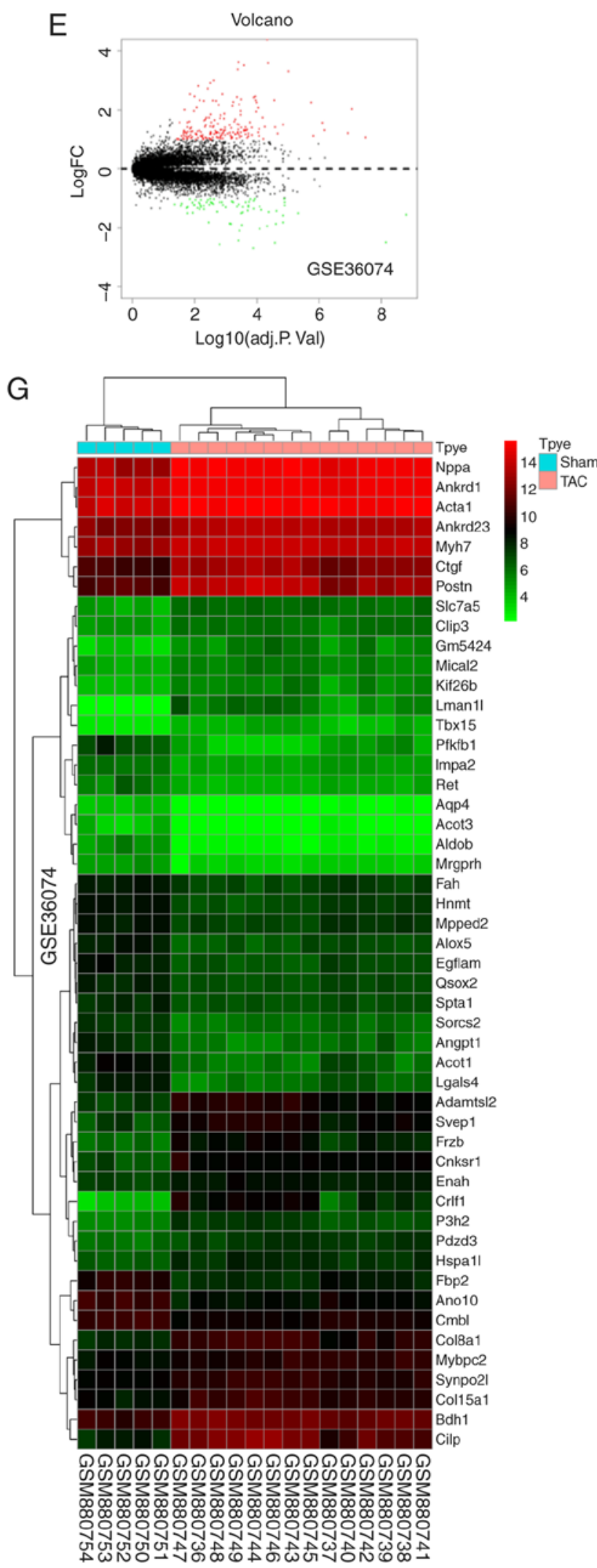
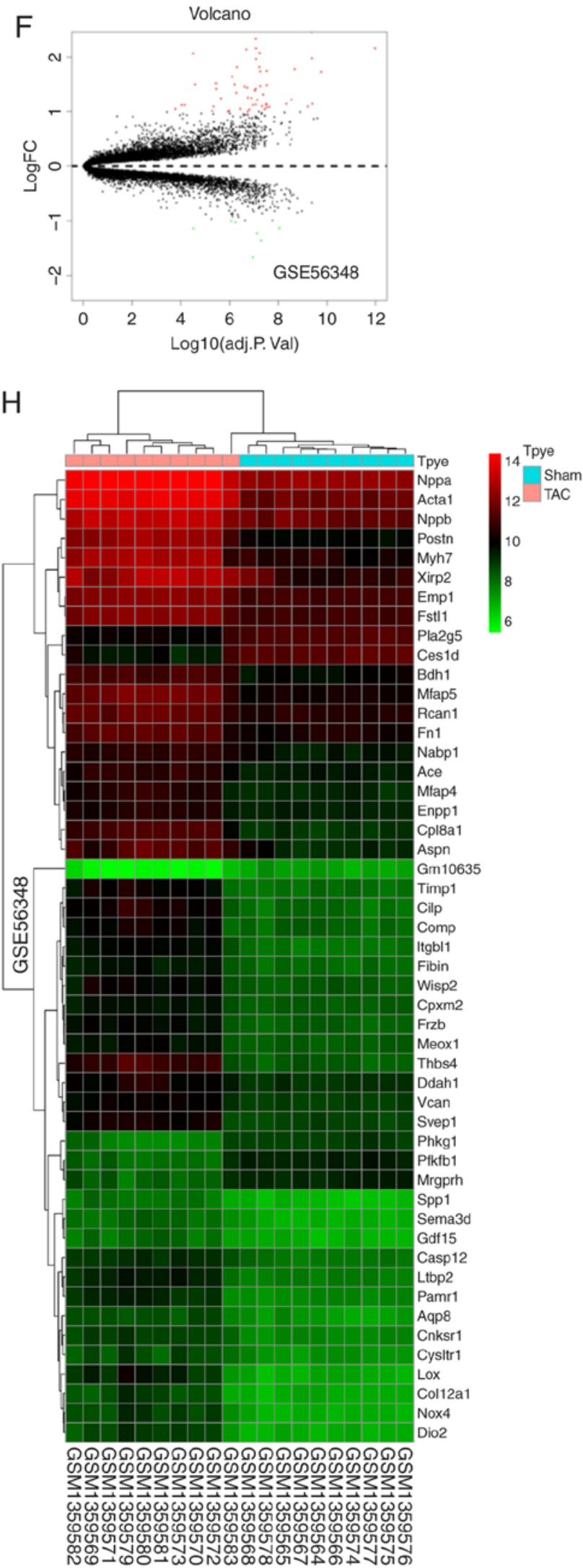

Figure 1. Continued. Volcano plot and heatmaps of differentially expressed genes (DEGs): (E) Volcano plot of DEGs for dataset GSE36074; (F) volcano plot of DEGs for dataset GSE56348; (G) heatmaps of DEGs for dataset GSE36074; (H) heatmaps of DEGs for dataset GSE56348.

phenotype, including increased HW/BW, LW/BW and HW/TL ratios (Fig. 4A-C). To investigate cardiac function, echocardiographic examination was performed. TAC mice exhibited deteriorated cardiac remodeling and dysfunction compared with sham-operated mice, as indicated by LVEDD, LVESD, FS and EF (Fig. 4D-H). H\&E staining and cardiomyocyte 
Table IV. Significant enriched analysis of differentially expressed genes.

\begin{tabular}{|c|c|c|c|c|}
\hline Category & Term & Description & Count & P-value \\
\hline GOTERM_BP_DIRECT & GO:0043206 & Extracellular fibril organization & 3 & 0.000070 \\
\hline GOTERM_BP_DIRECT & GO:0007155 & Cell adhesion & 6 & 0.000194 \\
\hline GOTERM_BP_DIRECT & GO:0042060 & Wound healing & 3 & 0.005265 \\
\hline GOTERM_BP_DIRECT & GO:0048251 & Elastic fiber assembly & 2 & 0.008103 \\
\hline GOTERM_BP_DIRECT & GO:0030308 & Negative regulation of cell growth & 3 & 0.009136 \\
\hline GOTERM_BP_DIRECT & GO:0061037 & Negative regulation of cartilage development & 2 & 0.009255 \\
\hline GOTERM_CC_DIRECT & GO:0005576 & Extracellular region & 20 & $<0.000001$ \\
\hline GOTERM_CC_DIRECT & GO:0005578 & Proteinaceous extracellular matrix & 11 & $<0.000000$ \\
\hline GOTERM_CC_DIRECT & GO:0005615 & Extracellular space & 15 & $<0.000000$ \\
\hline GOTERM_CC_DIRECT & GO:0031012 & Extracellular matrix & 9 & $<0.000000$ \\
\hline GOTERM_CC_DIRECT & GO:0005604 & Basement membrane & 4 & 0.000192 \\
\hline GOTERM_CC_DIRECT & GO:0070062 & Extracellular exosome & 9 & 0.008497 \\
\hline GOTERM_MF_DIRECT & GO:0008083 & Growth factor activity & 5 & 0.000016 \\
\hline GOTERM_MF_DIRECT & GO:0008201 & Heparin binding & 5 & 0.000019 \\
\hline GOTERM_MF_DIRECT & GO:0005509 & Calcium ion binding & 5 & 0.006123 \\
\hline
\end{tabular}

A

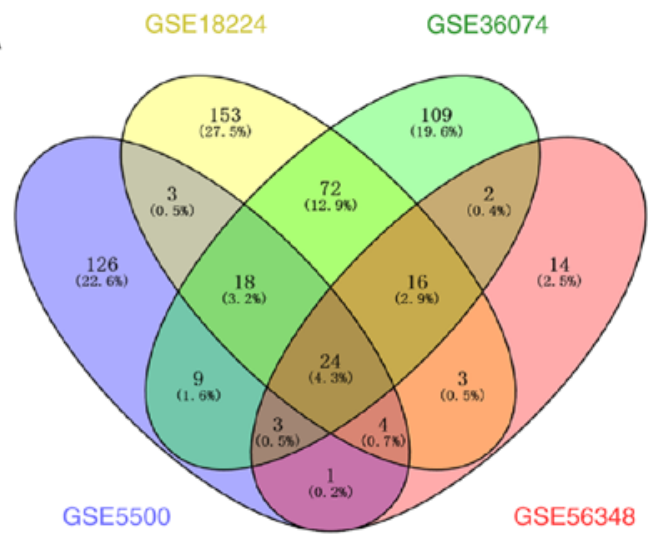

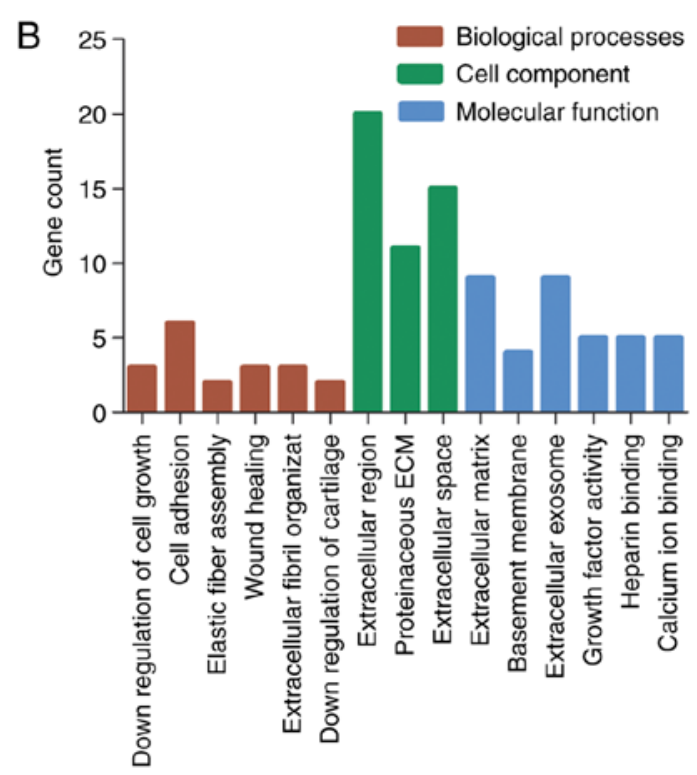

Figure 2. Identification of differentially expressed genes (DEGs) from the four data sets and Gene Ontology (GO) analysis. (A) Identification of 24 common DEGs from the four microarray datasets using venny website (http://bioinfogp.cnb.csic.es/tools/venny/index.html). Different color areas represented different microarray datasets. The cross-sectional areas reflect the commonly altered DEGs. Statistically significant DEGs were defined using $\mathrm{P}<0.05$ and $l \log \mathrm{FCl}>1$ as cut-offs. (B) DEG GO analysis classified the DEGs into 3 groups, namely molecular function, biological processes and cellular component.

cross-sectional area also confirmed the adverse effect of TAC surgery on cardiac remodeling (Fig. 4I and J). Several sections of the heart were stained with picrosirius red for assessment of interstitial fibrosis (Fig. 4K and L). Based on these results, the successful establishment of a TAC-induced cardiac pathological remodeling model in mice was confirmed.

Validation of gene expression levels of DEGs in a mouse model of cardiac remodeling. The mRNA expression levels of DEGs were detected using RT-PCR in a mouse model of TAC-induced cardiac remodeling, excluding the 9 published genes Postn, Lox, Ctgf, Nppa, ANP, Acta1, Timp1, Tgfb2 and
Clip, which have been previously confirmed to be directly involved in the occurrence and development of myocardial remodeling (these 9 genes have been found to be upregulated, which is consistent with the results of a large number of published studies) (22-30). Hence, the mRNA expression level of the remaining 16 DEGs was determined by RT-PCR. As shown in Fig. 5, compared with the sham group, the expression of Itgbl1, Aspn, Fstl1, Mfap5, Col8a1, Ltbp2, Mfap4, Pamr1, Cnksr1, Aqp8, Meox1, Gdf15 and Srpx was upregulated, and that of Retnla was downregulated in the mouse model of cardiac pathological remodeling, which is consistent with the results of the bioinformatics analysis. Frzb was not found 
A

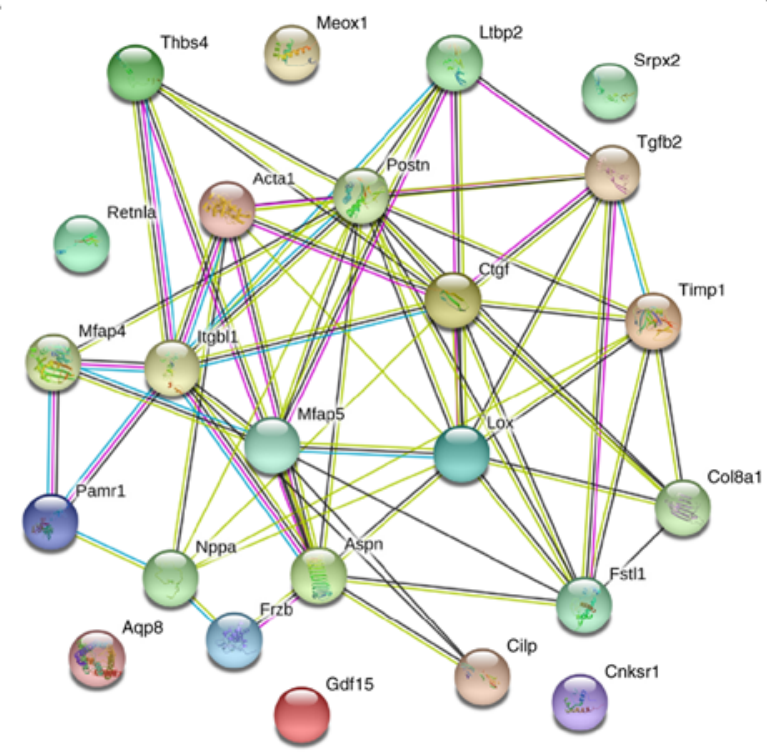

B

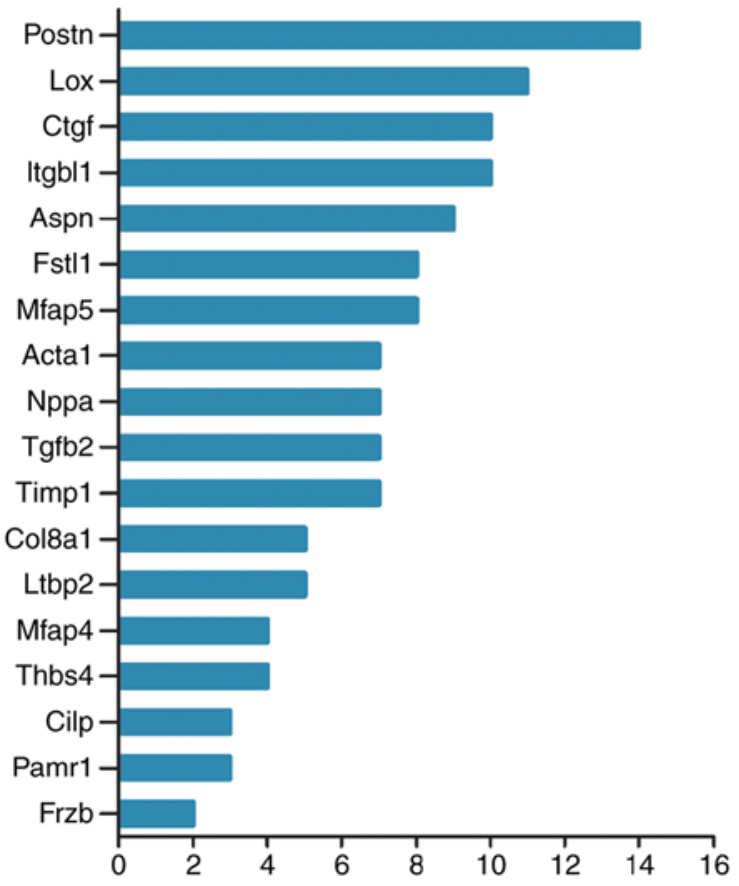

Figure 3. Differentially expressed gene (DEG) protein-protein interaction (PPI) network analysis. (A) Using the STRING online database, a total of 18 genes were filtered into the DEG PPI network complex. (B) Sorting the number of nodes of the 18 DEGs.

to be significantly differentially expressed, as determined by PCR. The reason for the inconsistency is that the sensitivity of different detection methods varies.

Detection of the protein level of key DEGs by western blotting and immunohistochemistry. According to the results of the PCR and combined with the hub genes by PPI network analysis, Itgbl1 and Aspn were selected, and their protein expression level was determined by western blotting and immunohistochemistry. As shown in Fig. 6, western blotting and immunohistochemistry confirmed that TAC surgery significantly increased the protein expression level of Itgbll and Asporin (protein encoded by Aspn gene), consistently with the results of PCR and bioinformatics analysis.

Expression of Itgbll and ASPN in CMs and CFs. CMs and CFs were extracted from neonatal rats, and stimulated by Ang II $(1 \mu \mathrm{M})$ and TGF- $\beta(10 \mathrm{ng} / \mathrm{ml})$, respectively. As shown in Fig. 7A and B, CMs were stained with anti- $\alpha$-actinin and CFs were stained with anti- $\alpha$-SMA. Ang II $(1 \mu \mathrm{M})$ increased the cross-sectional area of CMs (Fig. 7A and C), and TGF- $\beta$ $(10 \mathrm{ng} / \mathrm{ml})$ increased the fluorescence intensity of $\alpha$-SMA in CFs (Fig. 7B). The mRNA expression of Itgbll and ASPN in $\mathrm{CMs}$ and $\mathrm{CFs}$ was also detected. It was observed that Itgbl1 and ASPN were mainly expressed in CFs (Fig. 7D), and their expression was markedly increased following TGF- $\beta$ stimulation (Fig. 7E), while the expression of Itgbl1 and ASPN in CMs was very low, and there was no significant difference following stimulation by Ang II (Fig. 7F).

\section{Discussion}

In recent decades, ongoing research has unveiled that cardiac remodeling plays an important role in the pathophysiology of HF. In recent years, the emergence and rapid development of gene chip (microarray) and high-throughput sequencing technology has provided valuable targets for early detection, diagnosis, treatment and prognosis of various diseases, including HF. Comprehensive analysis of various microarray and high-throughput sequencing technologies using bioinformatics methods increases the sample size and reduces the impact of research error on the results, thus effectively improving their reliability.

In the present study, four microarray datasets (GSE5500, GSE18224, GSE36074 and GSE56348) were collected and downloaded for TAC-induced cardiac remodeling. These datasets were analyzed using limma package of $\mathrm{R}$ software, common DEGs (defined as $\log _{2} \mathrm{FC}>1$ or $\log _{2} \mathrm{FC}<-1$ and an adjusted P-value $<0.05$ ) were obtained, and 24 commonly altered DEGs (23 upregulated and 1 downregulated) were identified in the first step. Subsequently, these 24 DEGs were separated by GO terms into three groups, namely cellular component, molecular functions and biological process, using DAVID version 6.8. The result of GO term analysis indicated that these 24 DEGs datasets were mainly enriched in extracellular fibril organization, cell adhesion, wound healing, elastic fiber assembly, negative regulation of cell growth and cartilage development, extracellular region, proteinaceous extracellular matrix, extracellular space, extracellular matrix, basement membrane, extracellular exosome, growth factor activity, heparin binding and calcium ion binding. These functions are closely associated with the development of cardiac remodeling.

In the third step, a DEG PPI network complex was developed and a total of 18 DEGs (all upregulated) of the 24 commonly altered DEGs were filtered into this DEG complex, including (in descending order of the number of nodes) Postn, Lox, Ctgf, Itgbl1, Aspn, Fstl1, Mfap5, Acta1, Nppa, Tgfb2, Timp1, Col8a1, Ltbp2, Mfap4, Thbs4, Cilp, Pamr1 and Frzb. 
A

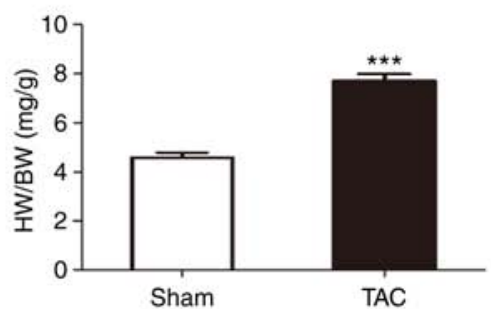

B

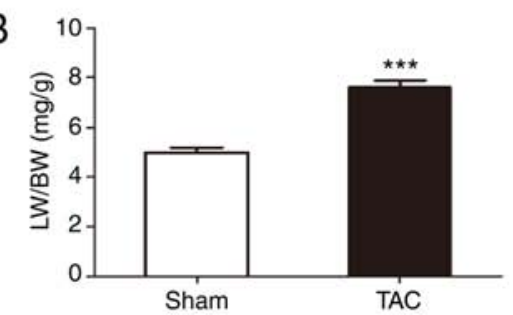

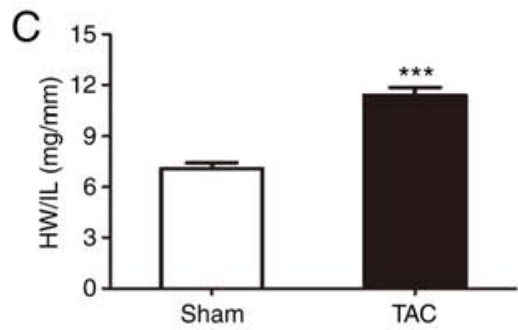

D

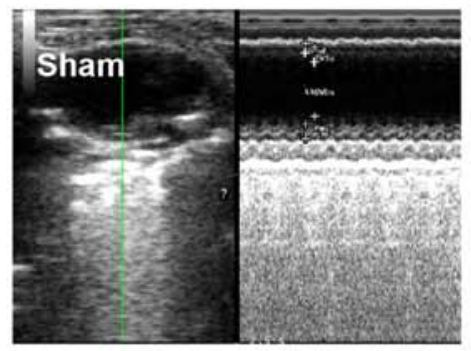

$\mathrm{F}$

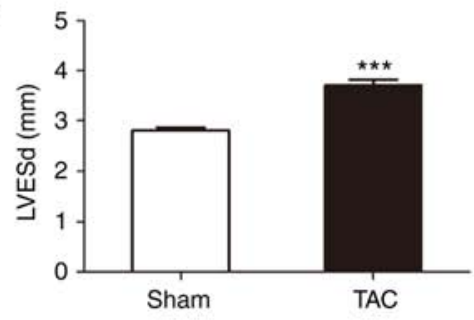

I

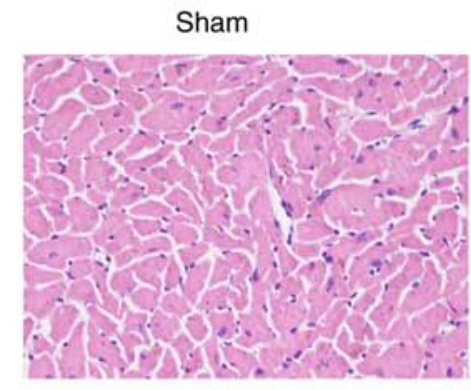

$\mathrm{K}$
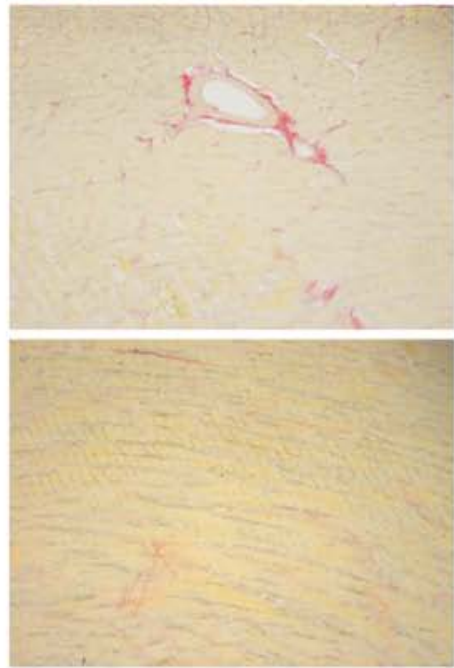

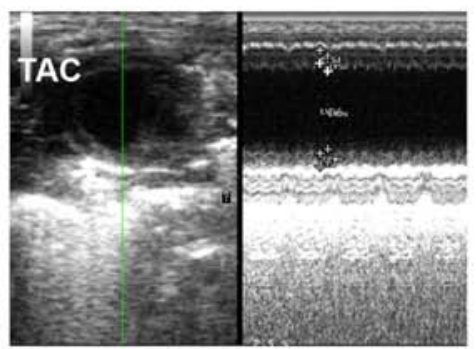

G

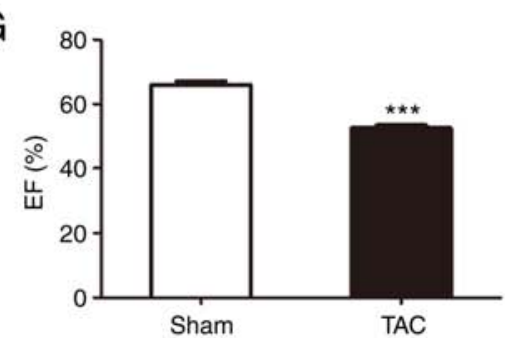

TAC
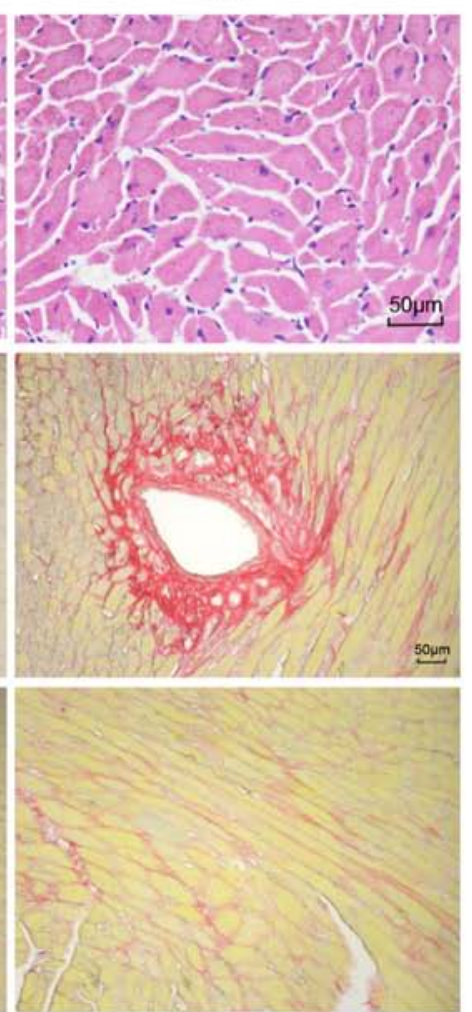
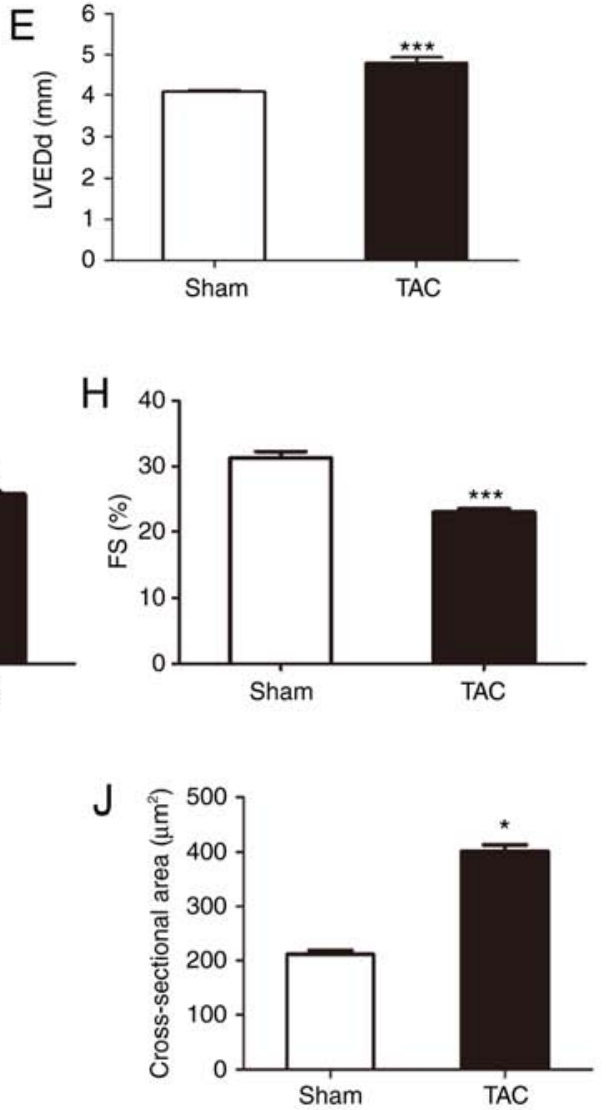

L

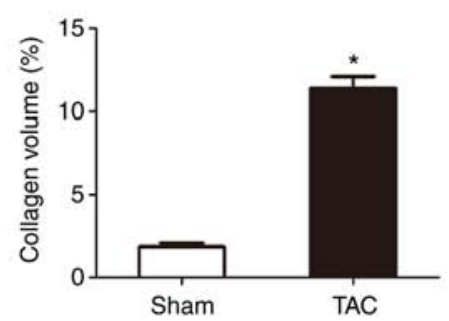

M

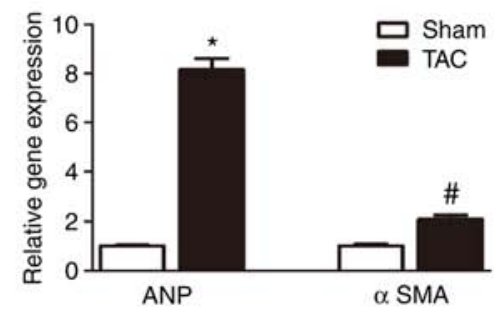

Figure 4. Established TAC-induced mouse model of cardiac remodeling. (A-C) Statistical results of the HW/BW, LW/BW and HW/TL ratios (n=11). (D-H) Echocardiography pictures and parameters (LVESD, LVEDD, EF and FS) (n=10). (I and J) Representative hematoxylin and eosin staining and statistical results for the cross-sectional area ( $\mathrm{n}=6$ sample, 100-150 cells per sample). $(\mathrm{K}$ and $\mathrm{L}$ ) Representative picrosirius red staining on histological sections and statistical results $(\mathrm{n}=6)$. (M) Reverse transcription-quantitative polymerase chain reaction analysis validation of mRNA expression levels of cardiac hypertrophy marker ANP and cardiac fibrosis marker $\alpha \mathrm{SMA}\left(\mathrm{n}=6\right.$ sample). ${ }^{*} \mathrm{P}<0.05,{ }^{*} \mathrm{P}<0.05,{ }^{* * *} \mathrm{P}<0.001$. TAC, transverse aortic constriction; HW, heart weight; BW, body weight; LW, lung weight; TL, tibial length; LVESD, left ventricular (LV) end-systolic diameter; LVEDD, LV end-diastolic dimension; EF, LV ejection fraction; FS, LV fractional shortening; ANP, atrial natriuretic peptide; SMA, smooth muscle actin. 
A

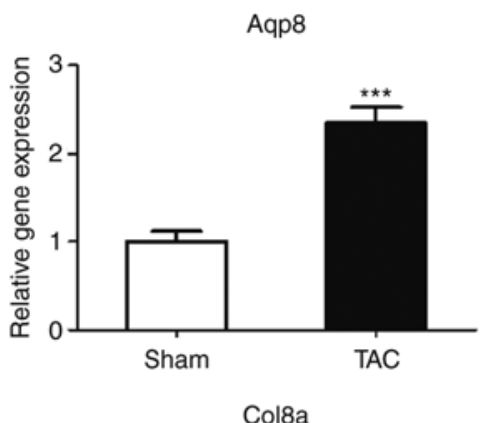

D

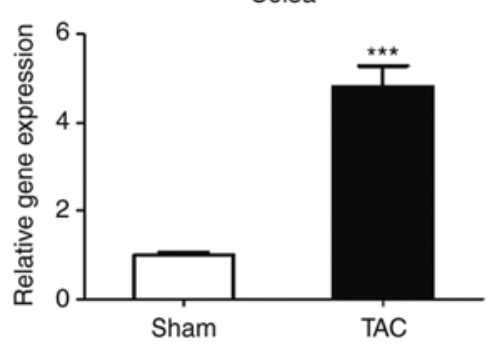

G

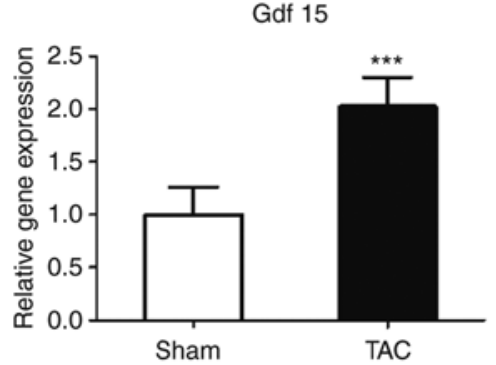

J

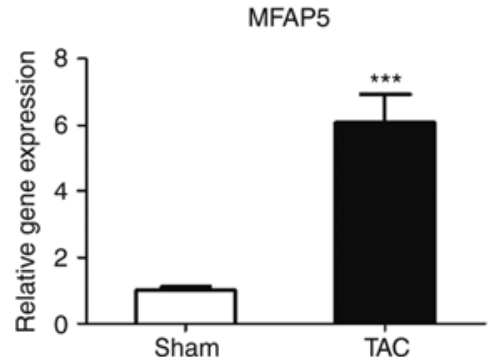

Pamr1

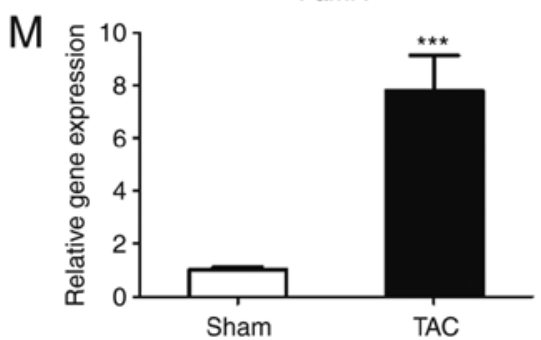

B

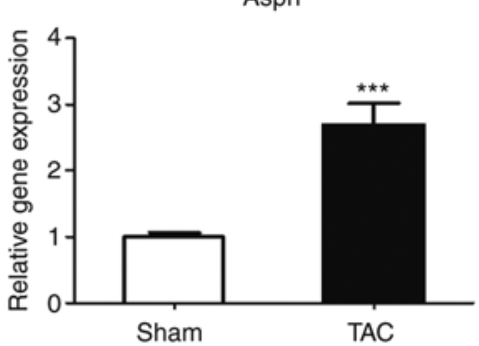

E

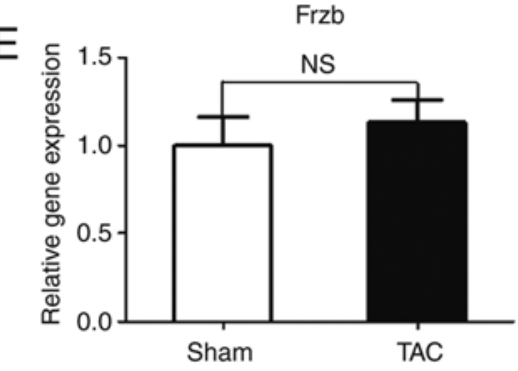

$\mathrm{H}$

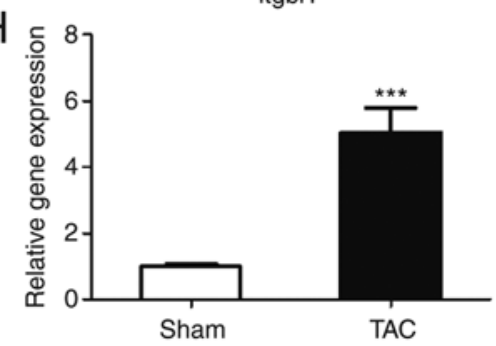

Meox1

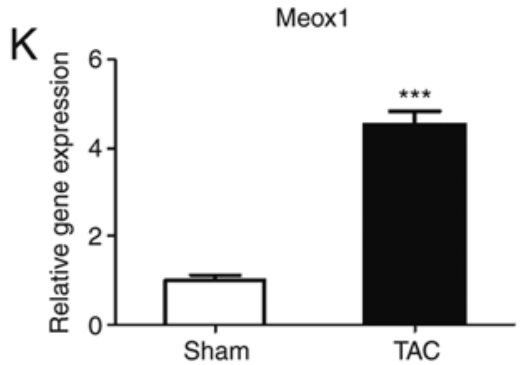

Renla

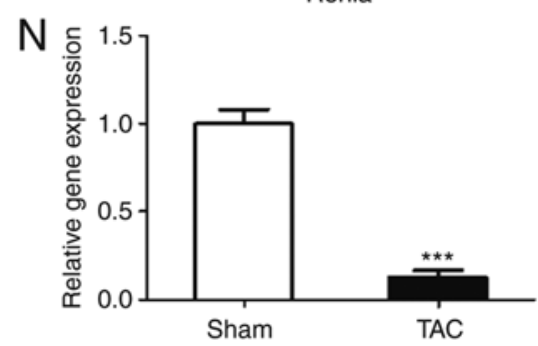

C

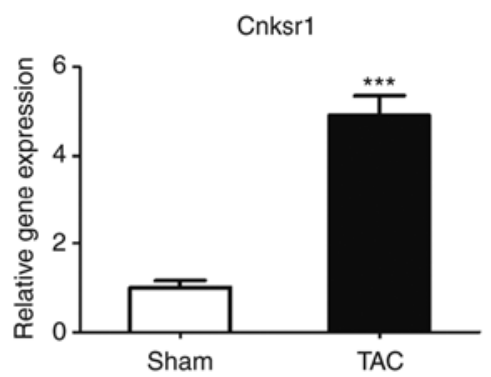

Fstl1

F

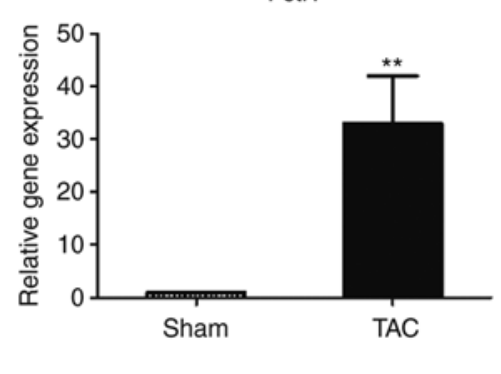

MFAP4

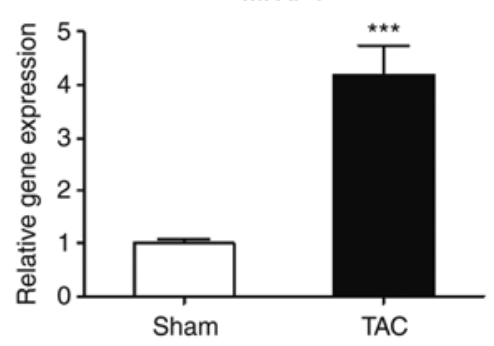

LTBP2

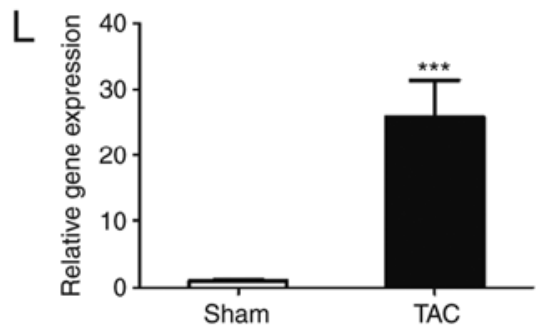

Srpx2

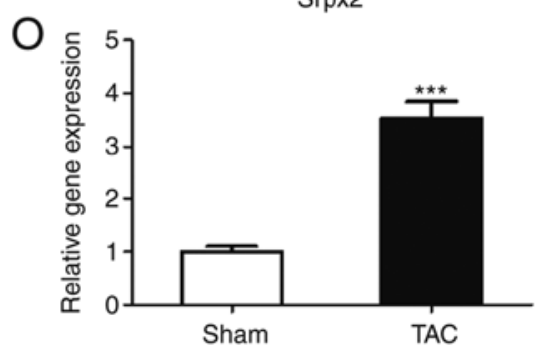

Figure 5. Reverse transcription-quantitative polymerase chain reaction analysis validation of mRNA expression levels of the selected common differentially expressed genes. (A-O) mRNA levels of Aqp8, Aspn, Cnksr1, Col8a1, Frzb, Fstl1, Gdf15, Itgbl1, Mfap4, Mfap5, Meox1, Ltbp2, Retnla and Pamr1 in heart tissues of mice in the TAC and sham-operated groups. Values shown are normalized to GAPDH $(\mathrm{n}=6) ;{ }^{* *} \mathrm{P}<0.01,{ }^{* * * *} \mathrm{P}<0.001$; NS, not significant; TAC, transverse aortic constriction.

Of these 24 DEGs, 9 (Postn, Lox, Ctgf, ANP, Acta1, Timp1, Tgfb2, Clip and Thbs4) have been previously reported to be directly involved in the development of cardiac remodeling (22-30); therefore, the level of expression of these genes was not examined in subsequent analyses. To verify the expression of the remaining 15 DEGs, a TAC-induced pressure overload model was constructed to elucidate the mechanism underlying cardiac remodeling. To verify whether the cardiac pathological remodeling model in mice was successfully established, echocardiography was used to detect cardiac function in the experimental and control groups, and gross morphological evaluation of the hearts was performed by H\&E staining. RT-PCR was used to verify that the expression of Itgbl1, Aspn, Fstl1, Mfap5, Col8a1, Ltbp2, Mfap4, Pamr1, Cnksr1, Aqp8, Meox1, Gdf15 and Srpx 
A

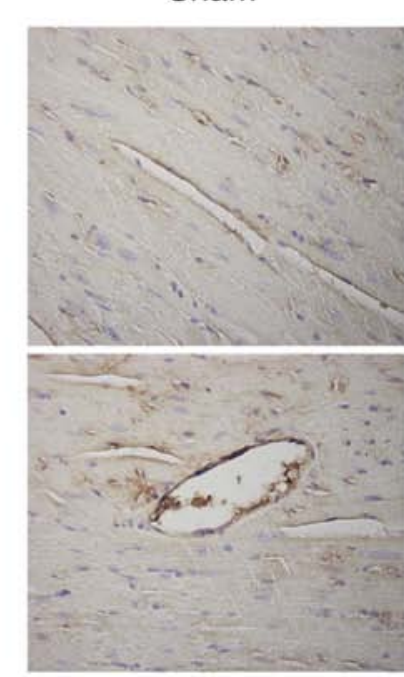

B
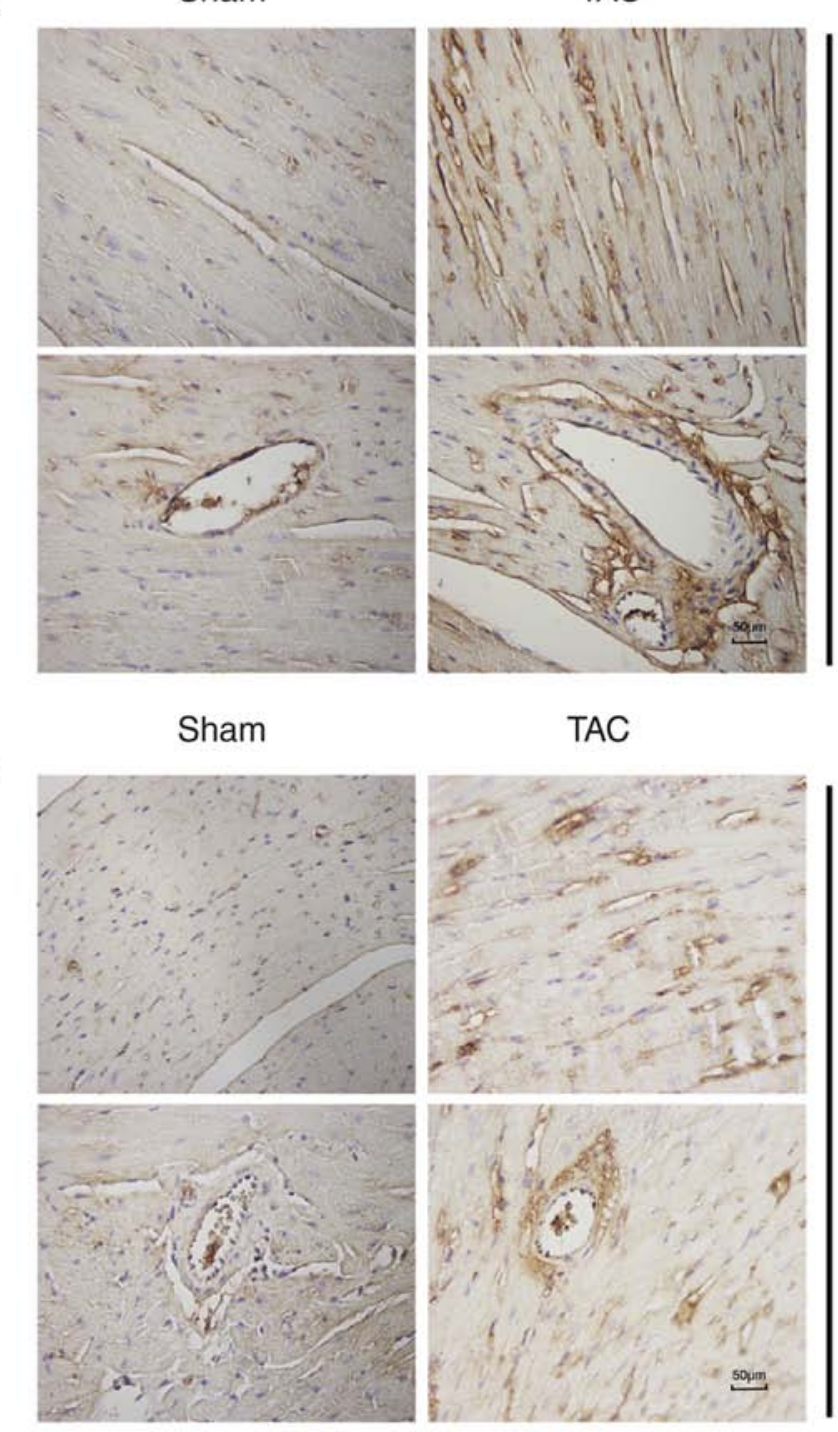

TAC

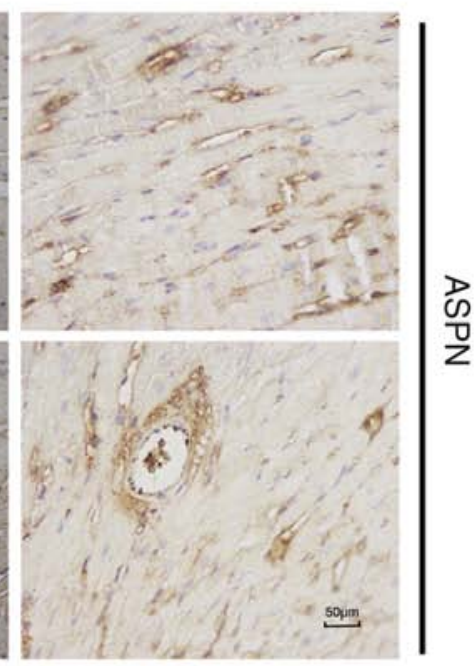

C
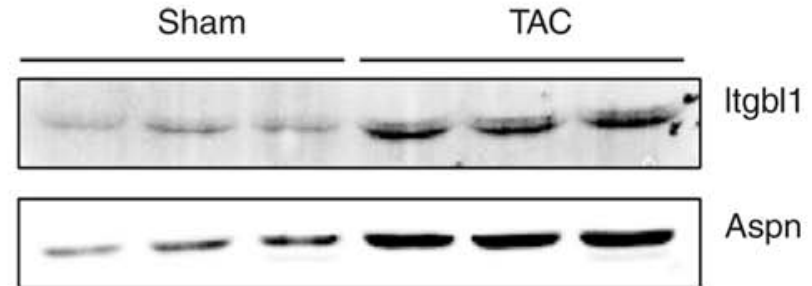

Aspn

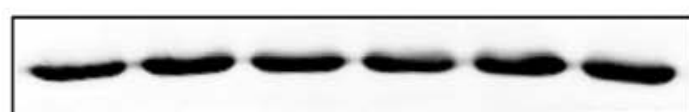

\section{GAPDH}

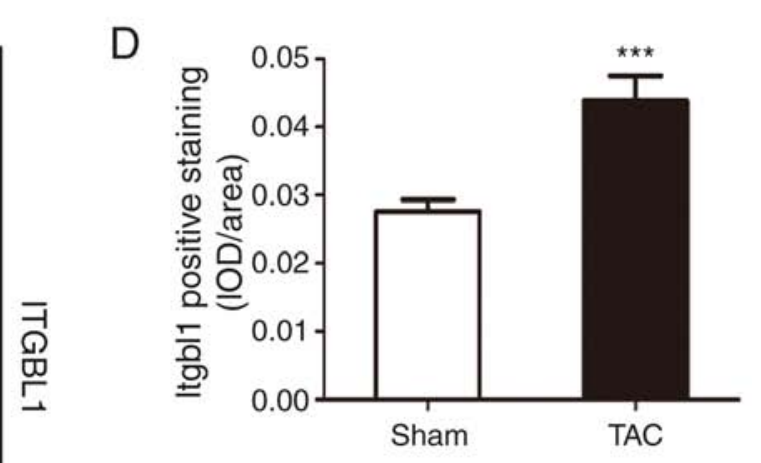

E

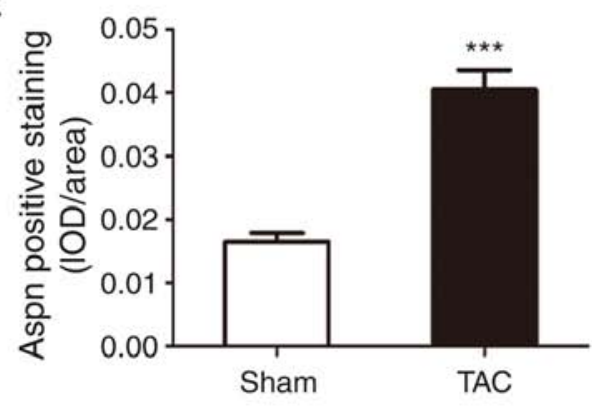

$\mathrm{F}$

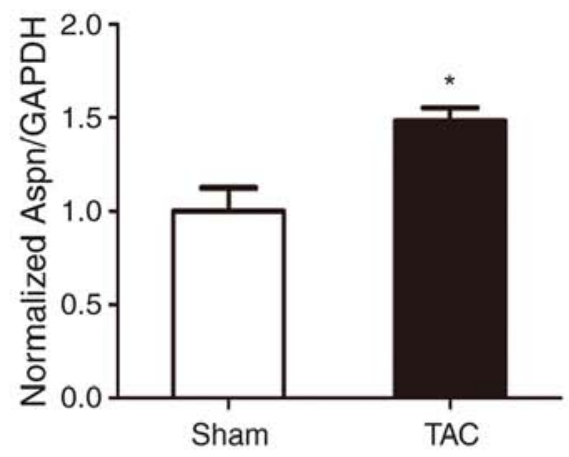

G

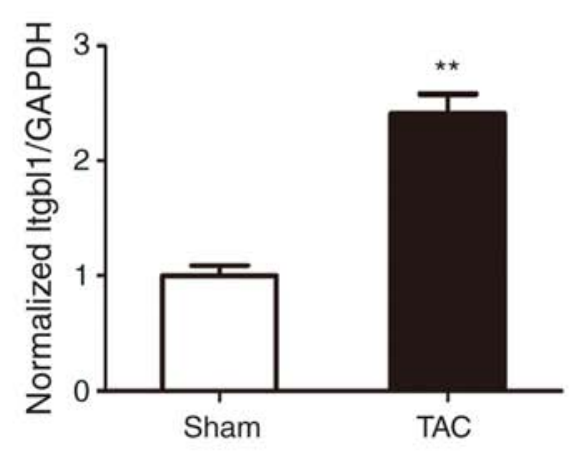

Figure 6. Western blotting and immunohistochemistry validation of protein expression levels of Itgbl1 and Aspn. (A) Immunohistochemistry images of protein expression of Itgbl1. (B) Immunohistochemistry images of protein expression of Itgbl1. (C) Western blots of protein expression of Itgbl1 and Aspn. (D-G) Immunohistochemistry and western blot bar graph $(\mathrm{n}=6)$. Values shown are normalized to GAPDH; ${ }^{*} \mathrm{P}<0.05,{ }^{* *} \mathrm{P}<0.01$, ${ }^{* * * *} \mathrm{P}<0.001 . \mathrm{ASPN}, \mathrm{Asporin}$; TAC, transverse aortic constriction.

was upregulated and that of Retnla was downregulated in the mouse model of cardiac pathological remodeling, which was consistent with the results of the bioinformatics analysis. Frzb was not significantly differentially expressed, as determined by RT-PCR. The reason for this inconsistency is that the sensitivity of different detection methods varies.
The Itgbl1 gene encodes a $\beta$-integrin-related protein. The Itgbl1 protein is an extracellular matrix protein that promotes ovarian cancer cell migration and adhesion through Wnt/PCP signaling and the FAK/SRC pathway (30). The Itgbl1 protein may promote bone metastasis of breast cancer by activating the TGF- $\beta$ signaling pathway (31). The Wnt/PCP, FAK/SRC 

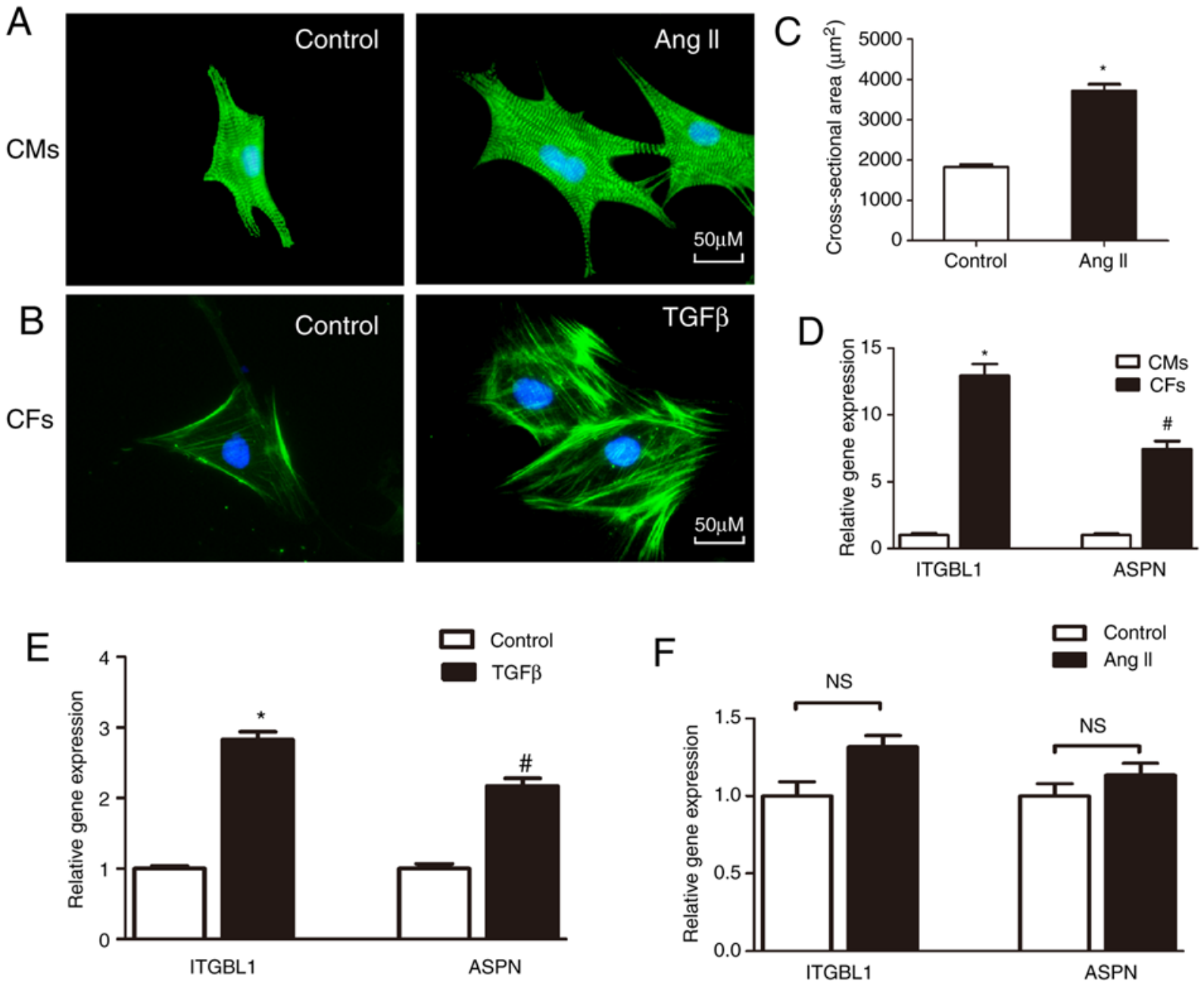

Figure 7. Expression of Itgbl1 and Asporin in cardiomyocytes (CMs) and cardiac fibroblasts (CFs). (A) Immunofluorescence staining of $\mathrm{CMs}$ with anti- $\alpha$-actinin (B) Immunofluorescence staining of CFs with anti- $\alpha$-SMA. (C) The cell surface area of CMs in the indicated groups ( $\mathrm{n}=6$ samples, with $100+$ cells per group), (D) Reverse transcription-quantitative polymerase chain reaction (RT-qPCR) analysis validation of mRNA expression levels of Itgbl1 and ASPN in CMs and CFs ( $\mathrm{n}=6$ sample). (E) RT-qPCR analysis validation of mRNA expression levels of Itgbl1 and ASPN in TGF $\beta$-treated CFs ( $\mathrm{n}=6$ sample). (F) RT-qPCR analysis validation of mRNA expression levels of Itgbll and ASPN in Ang II-treated CMs ( $\mathrm{n}=6$ sample) ${ }^{*} \mathrm{P}<0.05,{ }^{\prime \prime} \mathrm{P}<0.05$, NS, not significant. ASPN, Asporin; SMA, smooth muscle actin; TGF, transforming growth factor; Ang, angiotensin.

and TGF- $\beta$ signaling pathways play an important role in the process of cardiac remodeling. Similarly, Itgbl1 is a key regulator of fibrosis in patients with hepatitis $B$ virus-related liver fibrosis (32). This evidence suggests that Itgbll may be involved in the pathogenesis of cardiac remodeling. Aspn encodes Asporin, a cartilage extracellular protein that belongs to the small leucine-rich proteoglycan family. Asporin regulates chondrogenesis through regulating TGF- $\beta$ signaling; it can also bind to collagen and calcium and may induce collagen mineralization (33). There are currently no studies on the effects of Aspn on remodeling of various tissues.

The Fstll gene encodes a protein similar to follistatin. A previous study reported that the Fstll levels in a patient with asthma were associated with increased smooth muscle mass, and suggested that Fstll may contribute to airway remodeling in such patients (34). In addition, another study also confirmed that Fstl1 is involved in TGF- $\beta 1$-mediated pulmonary fibrosis (35). Fstll may also be used as a potential mediator of exercise-induced cardioprotection post-myocardial infarction (36). However, there has yet been no study that elucidates whether Fstll participates in pressure overload-mediated cardiac remodeling.
The microfibril-associated protein (Mfap) 4 and Mfap5 genes encode extracellular matrix proteins that are involved in cell adhesion and intercellular interaction. A number of studies have confirmed the involvement of Mfap4 in vascular remodeling after vascular injury and airway remodeling induced by bronchial asthma $(37,38)$. Furthermore, the expression of Mfap4 in the serum and liver of patients with liver cirrhosis was found to be significantly increased (39). Mfap5 is associated with obesity-associated adipose tissue and extracellular matrix remodeling and regulates adipose tissue inflammation (40).

Collagen type VIII alpha 1 chain (Col8a1) encodes one of the two $\alpha$ chains of type VIII collagen, which is a key component of the basement membrane of the corneal endothelium. A previous study demonstrated that Col8a1 mediates vessel wall remodeling following arterial injury and fibrous cap formation in atherosclerosis. The latent transforming growth factor beta binding protein 2 (Ltbp2) gene encodes a protein that belongs to the latent TGF- $\beta$-binding protein (LTBP) family, and this protein shares similarities with the fibrillins. Ltbp2 is a potential prognostic blood biomarker that may reflect the level of differentiation of lung fibroblasts into myofibroblasts in idiopathic 
pulmonary fibrosis (41). Peptidase domain containing associated with muscle regeneration 1 (Pamr1) is a putative tumor suppressor that is frequently inactivated by promoter hypermethylation in breast cancer tissues (42). However, there is little research on Pamrl, so its role remains elusive.

The connector enhancer of kinase suppressor of Ras 1 (Cnksr1) gene encodes a protein containing several motifs involved in PPI. The Cnksrl protein can mediate the association among different signaling pathways (43). Similar to the Pamrl gene, there is little research on the exact role of the Cnksrl gene. The aquaporin 8 (Aqp8) gene encodes a protein that belongs to a family of small integral membrane proteins, and functions as a water channel (44). The sushi repeat-containing protein X-linked (Srpx) gene encodes the Srpx protein, which is involved in phagocytosis during disk shedding, cell adhesion to cells other than the pigment epithelium, or signal transduction (45). At present, it remains unknown whether Aqp8 and Srpx are involved in the remodeling of cardiac or other tissues. The mesenchyme homeobox 1 (Meox1) gene encodes a member of a subfamily of antennapedia-like homeobox-containing proteins. Meox1 is part of a regulatory circuit that serves an essential, non-redundant function in the maintenance of rostro-caudal sclerotome polarity and leads to remodeling of the cranio-cervical joints of the axial skeleton (46). Meox1 promotes vascular smooth muscle cell phenotypic modulation and balloon injury-induced vascular remodeling via regulating the FAK-ERK1/2 signaling cascade (47).

The growth differentiation factor 15 (Gdf15) gene encodes a secreted ligand of the TGF- $\beta$ superfamily of proteins, which bind various TGF- $\beta$ receptors (TGF- $\beta$ R) and leads to activation of Smad family transcription factors. The TGF- $\beta / \mathrm{Smad}$ signaling pathway is closely associated with cardiac remodeling. Resistin-like alpha (Retnla) is also referred to as hypoxia-induced mitogenic factor (Himf). In a model of lung inflammation, Retnla knockout led to lung inflammation and higher Th2 cell cytokine production compared with that observed in wild-type mice. Retnla strongly activates Akt phosphorylation, and treatment with Retnla has been shown to result in a significant reduction of apoptosis in cultured embryonic lung cells $(48,49)$. Retnla is involved in immune response-induced pulmonary vascular remodeling and enhanced inflammatory response typically observed after intermittent ovalbumin challenge (50).

There were certain limitations to the present study. First, the amount of data obtained from the GEO database is not sufficiently comprehensive, as some of the data samples are too small to screen for common DEGs, and were thus excluded. Second, DEGs represent only one type of molecular changes that occur in the mouse model of TAC-induced cardiac remodeling. Other factors, including non-coding RNAs (such as microRNAs, long non-coding RNAs and circular RNAs), gene mutations and metabolomic changes, should also be considered to provide a comprehensive understanding of the development of cardiac remodeling. Third, of the 15 DEGs, only the protein levels of Itgbl1 and Asporin were verified by western blotting and immunohistochemistry. Fourth, the findings of the present study only indicated that the expression of some genes was altered during cardiac remodeling, but did not elucidate the underlying mechanism. This study is only part of our research group on HF. Through bioinformatics analysis, the DEGs were screened and preliminarily verified. By screening the candidate genes, related gene knockout and overexpression mouse models are constructed in order to provide a solid early basis for exploring the pathogenesis and treatment of HF in the future.

In conclusion, the present study identified DEGs in mouse hearts with TAC-induced cardiac remodeling and sham samples by bioinformatics analysis, thereby indicating the crucial role of DEGs in the process of cardiac remodeling. The study findings provide a set of candidate genes for future investigation of the mechanisms and selection of biomarkers for cardiac remodeling. In future research, knockout or transgenic mice will be used to further elucidate the pathophysiological mechanisms underlying the involvement of these genes in cardiac remodeling in vivo and in vitro.

\section{Acknowledgements}

Not applicable.

\section{Funding}

The present was supported by grants from the National Natural Science Foundation of China (no. 81470516) and the Key Project of the National Natural Science Foundation (no. 81530012).

\section{Availability of data and materials}

The datasets generated and/or analyzed during the present study are available from the corresponding author on reasonable request. The URLs to all datasets used in this study as follows: GSE5500 datasets: https://www.ncbi. nlm.nih.gov/sites/GDSbrowser?acc=GDS2316; GSE18224 datasets: https://www.ncbi.nlm.nih.gov/geo/query/acc. cgi?acc=GSE18224; GSE36074 datasets: https:/www.ncbi. nlm.nih.gov/geo/query/acc.cgi?acc=GSE36074; GSE56348 datasets: https://www.ncbi.nlm.nih.gov/geo/query/acc. cgi?acc=GSE56348.

\section{Authors' contributions}

HBW and QZT conceived and designed the study; HBW, KY and RH performed data analysis; MX, DF, MXL and LBL performed the experiments; $\mathrm{SHH}$ and HMW wrote the paper. All the authors have read and approved the final version of this manuscript for publication.

\section{Ethics approval and consent to participate}

All experiments have been approved by the Ethics Committee of The Renmin Hospital of Wuhan University (Wuhan, China).

\section{Patient consent for publication.}

Not applicable.

\section{Competing interests}

The authors declare that they have no competing interests. 


\section{References}

1. Yusuf S, Wood D, Ralston J and Reddy KS: The World Heart Federation's vision for worldwide cardiovascular disease prevention. Lancet 386: 399-402, 2015.

2. Sandri M, Viehmann M, Adams V, Rabald K, Mangner N, Höllriegel R, Lurz P, Erbs S, Linke A, Kirsch K, et al: Chronic heart failure and aging-effects of exercise training on endothelial function and mechanisms of endothelial regeneration: Results from the leipzig exercise intervention in chronic heart failure and aging (LEICA) study. Eur J Prev Cardiol 23: 349-358, 2016.

3. Schirone L, Forte M, Palmerio S, Yee D, Nocella C, Angelini F, Pagano F, Schiavon S, Bordin A, Carrizzo A, et al: A review of the molecular mechanisms underlying the development and progression of cardiac remodeling. Oxid Med Cell Longev 2017: 3920195, 2017.

4. Wu QQ, Xiao Y, Yuan Y, Ma ZG, Liao HH, Liu C, Zhu JX Yang Z, Deng W and Tang QZ: Mechanisms contributing to cardiac remodelling. Clin Sci (Lond) 131: 2319-2345, 2017.

5. Keeler AM and Flotte TR: Cell and gene therapy for genetic diseases: Inherited disorders affecting the lung and those mimicking sudden infant death syndrome. Hum Gene Ther 23: 548-556, 2012.

6. Barbosa C, Peixeiro I and Romao L: Gene expression regulation by upstream open reading frames and human disease. PLoS Genet 9: e1003529, 2013.

7. Patel A and Cheung SW: Application of DNA Microarray to clinical diagnostics. Methods Mol Biol 1368: 111-132, 2016.

8. Hanif W, Alex L, Su Y, Shinde AV, Russo I, Li N and Frangogiannis NG: Left atrial remodeling, hypertrophy, and fibrosis in mouse models of heart failure. Cardiovasc Pathol 30 27-37, 2017

9. Guo Y, Bao Y, Ma M and Yang W: Identification of key candidate genes and pathways in colorectal cancer by integrated bioinformatical analysis. Int J Mol Sci 18: E722, 2017.

10. Bisping E, Ikeda S, Kong SW, Tarnavski O, Bodyak N, McMullen JR, Rajagopal S, Son JK, Ma Q, Springer Z, et al: Gata4 is required for maintenance of postnatal cardiac function and protection from pressure overload-induced heart failure. Proc Natl Acad Sci USA 103: 14471-14476, 2006.

11. Fliegner D, Schubert C, Penkalla A, Witt H, Kararigas G, Dworatzek E, Staub E, Martus P, Ruiz Noppinger P, Kintscher U, et al: Female sex and estrogen receptor-beta attenuate cardiac remodeling and apoptosis in pressure overload. Am J Physiol Regul Integr Comp Physiol 298: R1597-R1606, 2010.

12. Skrbic B, Bjørnstad JL, Marstein HS, Carlson CR, Sjaastad I, Nygård S, Bjørnstad S, Christensen $\mathrm{G}$ and Tønnessen T: Differential regulation of extracellular matrix constituents in myocardial remodeling with and without heart failure following pressure overload. Matrix Biol 32: 133-142, 2013.

13. Lai L, Leone TC, Keller MP, Martin OJ, Broman AT, Nigro J, Kapoor K, Koves TR, Stevens R, Ilkayeva OR, et al: Energy metabolic reprogramming in the hypertrophied and early stage failing heart: A multisystems approach. Circ Heart Fail 7 : 1022-1031, 2014

14. Huang da W, Sherman BT and Lempicki RA: Systematic and integrative analysis of large gene lists using DAVID bioinformatics resources. Nat Protoc 4: 44-57, 2009.

15. Popik OV, Saik OV, Petrovskiy ED, Sommer B, Hofestädt R, Lavrik IN and Ivanisenko VA: Analysis of signaling networks distributed over intracellular compartments based on protein-protein interactions. BMC Genomics 15 (Suppl 12): S7, 2014.

16. Yuan YP, Ma ZG, Zhang X, Xu SC, Zeng XF, Yang Z, Deng W and Tang QZ: CTRP3 protected against doxorubicin-induced cardiac dysfunction, inflammation and cell death via activation of Sirt1. J Mol Cell Cardiol 114: 38-47, 2018.

17. Ma ZG, Dai J, Zhang WB, Yuan Y, Liao HH, Zhang N, Bian ZY and Tang QZ: Protection against cardiac hypertrophy by geniposide involves the GLP-1 receptor/AMPK $\alpha$ signalling pathway. $\mathrm{Br}$ J Pharmacol 173: 1502-1516, 2016.

18. Zhang X, Ma ZG, Yuan YP, Xu SC, Wei WY, Song P, Kong CY, Deng W and Tang QZ: Rosmarinic acid attenuates cardiac fibrosis following long-term pressure overload via AMPK $\alpha / \operatorname{Smad} 3$ signaling. Cell Death Dis 9: 102, 2018.

19. Wang HB, Yang J, Ding JW, Chen LH, Li S, Liu XW, Yang CJ, Fan ZX and Yang J: RNAi-mediated downregulation of CD47 protects against ischemia/reperfusion-induced myocardial damage via activation of eNOS in a rat model. Cell Physiol Biochem 40: 1163-1174, 2016.
20. Schorb W, Booz GW, Dostal DE, Conrad KM, Chang KC and Baker KM: Angiotensin II is mitogenic in neonatal rat cardiac fibroblasts. Circ Res 72: 1245-1254,1993.

21. Toth A, Jeffers JR, Nickson P, Min JY, Morgan JP, Zambetti GP and Erhardt P: Targeted deletion of Puma attenuates cardiomyocyte death and improves cardiac function during ischemia-reperfusion. Am J Physiol Heart Circ Physiol 291: H52-H60, 2006.

22. Azhar M, Brown K, Gard C, Chen H, Rajan S, Elliott DA, Stevens MV, Camenisch TD, Conway SJ and Doetschman T: Transforming growth factor Beta2 is required for valve remodeling during heart development. Dev Dyn 240: 2127-2141, 2011.

23. Takawale A, Zhang P, Patel VB, Wang X, Oudit G and Kassiri Z: Tissue inhibitor of matrix metalloproteinase-1 promotes myocardial fibrosis by mediating CD63-integrin $\beta 1$ interaction. Hypertension 69: 1092-1103, 2017.

24. Schwartz K, de la Bastie D, Bouveret P, Oliviéro P, Alonso S and Buckingham M: Alpha-skeletal muscle actin mRNA's accumulate in hypertrophied adult rat hearts. Circ Res 59: 551-555, 1986.

25. Deschepper CF, Masciotra S, Zahabi A, Boutin-Ganache I, Picard S and Reudelhuber TL: Functional alterations of the Nppa promoter are linked to cardiac ventricular hypertrophy in WKY/WKHA rat crosses. Circ Res 88: 223-228, 2001.

26. Liu X, Gai Y, Liu F, Gao W, Zhang Y, Xu M and Li Z: Trimetazidine inhibits pressure overload-induced cardiac fibrosis through NADPH oxidase-ROS-CTGF pathway. Cardiovasc Res 88: 150-158, 2010.

27. Hu C, Dandapat A, Chen J, Fujita Y, Inoue N, Kawase Y, Jishage K, Suzuki H, Sawamura T and Mehta JL: LOX-1 deletion alters signals of myocardial remodeling immediately after ischemia-reperfusion. Cardiovasc Res 76: 292-302, 2007.

28. Kaur H, Takefuji M, Ngai CY, Carvalho J, Bayer J, Wietelmann A, Poetsch A, Hoelper S, Conway SJ, Möllmann H, et al: Targeted ablation of periostin-expressing activated fibroblasts prevents adverse cardiac remodeling in mice. Circ Res 118: 1906-1917, 2016

29. Zhang CL, Zhao Q, Liang H, Qiao X, Wang JY, Wu D, Wu LL and Li L: Cartilage intermediate layer protein-1 alleviates pressure overload-induced cardiac fibrosis via interfering TGF- $\beta 1$ signaling. J Mol Cell Cardiol 116: 135-144, 2018.

30. Sun L, Wang D, Li X, Zhang L, Zhang H and Zhang Y: Extracellular matrix protein ITGBL1 promotes ovarian cancer cell migration and adhesion through Wnt/PCP signaling and FAK/SRC pathway. Biomed Pharmacother 81: 145-151, 2016.

31. Li XQ, Du X, Li DM, Kong PZ, Sun Y, Liu PF, Wang QS and Feng YM: ITGBL1 is a Runx2 transcriptional target and promotes breast cancer bone metastasis by activating the TGF $\beta$ signaling pathway. Cancer Res 75: 3302-3313, 2015.

32. Wang M, Gong Q, Zhang J, Chen L, Zhang Z, Lu L, Yu D, Han Y, Zhang D, Chen $\mathrm{P}$, et al: Characterization of gene expression profiles in HBV-related liver fibrosis patients and identification of ITGBL1 as a key regulator of fibrogenesis. Sci Rep 7: 43446, 2017.

33. Kaliakatsos M, Tzetis M, Kanavakis E, Fytili P, Chouliaras G, Karachalios T, Malizos K and Tsezou A: Asporin and knee osteoarthritis in patients of Greek origin. Osteoarthritis Cartilage 14: 609-611, 2006.

34. Liu Y, Liu T, Wu J, Li T, Jiao X, Zhang H, Zhao J, Wang J, Liu L, Cao L, et al: The Correlation between FSTL1 expression and airway remodeling in asthmatics. Mediators Inflamm 2017: 7918472, 2017

35. Zheng X, Qi C, Zhang S, Fang Y and Ning W: TGF- $\beta 1$ induces Fstl1 via the Smad3-c-Jun pathway in lung fibroblasts. Am J Physiol Lung Cell Mol Physiol 313: L240-L251, 2017.

36. Xi Y, Gong DW and Tian Z: FSTL1 as a potential mediator of exercise-induced cardioprotection in post-myocardial infarction rats. Sci Rep 6: 32424, 2016.

37. Schlosser A, Pilecki B, Hemstra LE, Kejling K, Kristmannsdottir GB, Wulf-Johanss on H, Moeller JB, Füchtbauer EM, Nielsen O, Kirketerp-Møller K, et al: MFAP4 promotes vascular smooth muscle migration, proliferation and accelerates neointima formation. Arterioscler Thromb Vasc Biol 36: 122-133, 2016.

38. Pilecki B, Schlosser A, Wulf-Johansson H, Trian T, Moeller JB, Marcussen N, Aguilar-Pimentel JA, de Angelis MH, Vestbo J, Berger P, et al: Microfibrillar-associated protein 4 modulates airway smooth muscle cell phenotype in experimental asthma. Thorax 70: 862-872, 2015.

39. Mölleken C, Sitek B, Henkel C, Poschmann G, Sipos B, Wiese S, Warscheid B, Broelsch C, Reiser M, Friedman SL, et al: Detection of novel biomarkers of liver cirrhosis by proteomic analysis. Hepatology 49: 1257-1266, 2009. 
40. Vaittinen M, Kolehmainen M, Ryden M, Eskelinen M, Wabitsch M, Pihlajamäki J, Uusitupa M and Pulkkinen L: MFAP5 is related to obesity-associated adipose tissue and extracellular matrix remodeling and inflammation. Obesity (Silver Spring) 23: 1371-1378, 2015.

41. Enomoto Y, Matsushima S, Shibata K, Aoshima Y, Yagi H, Meguro S, Kawasaki H, Kosugi I, Fujisawa T, Enomoto N, et al: LTBP2 is secreted from lung myofibroblasts and is a potential biomarker for idiopathic pulmonary fibrosis. Clin Sci (Lond) 132: $1565-1580,2018$

42. Lo PH, Tanikawa C, Katagiri T, Nakamura Y and Matsuda K: Identification of novel epigenetically inactivated gene PAMR1 in breast carcinoma. Oncol Rep 33: 267-273, 2015.

43. Fischer A, Muhlhauser WWD, Warscheid B and Radziwill G: Membrane localization of acetylated CNK1 mediates a positive feedback on RAF/ERK signaling. Sci Adv 3: e1700475, 2017.

44. Bestetti S, Medraño-Fernandez I, Galli M, Ghitti M, Bienert GP, Musco G, Orsi A, Rubartelli A and Sitia R: A persulfidation-based mechanism controls aquaporin-8 conductance. Sci Adv 4: eaar5770, 2018.

45. Meindl A, Carvalho MR, Herrmann K, Lorenz B, Achatz H, Lorenz B, Apfelstedt-Sylla E, Wittwer B, Ross M and Meitinger T: A gene (Srpx) encoding a sushi-repeat-containing protein is deleted in patients with X-linked retinitis-pigmentosa. Hum Mol Genet 4: 2339-2346, 1995.

46. Skuntz S, Mankoo B, Nguyen MT, Hustert E, Nakayama A Tournier-Lasserve E, Wright CV, Pachnis V, Bharti K and Arnheiter H: Lack of the mesodermal homeodomain protein MEOX1 disrupts sclerotome polarity and leads to a remodeling of the cranio-cervical joints of the axial skeleton. Dev Biol 332: 383-395, 2009
47. Wu B, Zhang L, Zhu YH, Zhang YE, Zheng F, Yang JY, Guo LY, Li XY, Wang L, Tang JM, et al: Mesoderm/mesenchyme homeobox gene 1 promotes vascular smooth muscle cell phenotypic modulation and vascular remodeling. Int J Cardiol 251: 82-89, 2018.

48. Pesce JT, Ramalingam TR, Wilson MS, Mentink-Kane MM, Thompson RW, Cheever AW, Urban JF Jr and Wynn TA: Retnla (relmalpha/fizz1) suppresses helminth-induced Th2-type immunity. PLoS Pathog 5: e1000393, 2009.

49. Nair MG, Du Y, Perrigoue JG, Zaph C, Taylor JJ, Goldschmidt M, Swain GP, Yancopoulos GD, Valenzuela DM, Murphy A, et al: Alternatively activated macrophage-derived RELM-\{alpha\} is a negative regulator of type 2 inflammation in the lung. $J$ Exp Med 206: 937-952, 2009.

50. Angelini DJ,Su Q, Yamaji-Kegan K, Fan C,Skinner JT, Poloczek A, El-Haddad H, Cheadle C and Johns RA: Hypoxia-induced mitogenic factor $(\mathrm{HIMF} / \mathrm{FIZZ1/RELM} \alpha$ ) in chronic hypoxia- and antigen-mediated pulmonary vascular remodeling. Respir Res 14: $1,2013$.

This work is licensed under a Creative Commons Attribution-NonCommercial-NoDerivatives 4.0 International (CC BY-NC-ND 4.0) License. 